\title{
Effects of Confinement on Bubble Dynamics in a Square Duct
}

\author{
Purushotam Kumar*and Surya P. Vanka ${ }^{\dagger}$ \\ Department of Mechanical Science and Engineering, University of Illinois at \\ Urbana-Champaign
}

May 16, 2015

\begin{abstract}
In this paper, we simulate the effects of confinement on three-dimensional bubble dynamics in a square duct. A systematic study of three confinement ratios, four Bond numbers and two Morton numbers has been conducted. A GPU implemented VOF numerical algorithm on a Cartesian collocated grid with an improved method to handle the surface tension force has been developed. A three-dimensional geometry construction method with a piecewise linear interpolation scheme for approximating the interface segments is used to compute the interface shape and liquid volume flux from the cell faces. The Navier-Stokes equations are solved with a space-time second-order accurate fractional step numerical scheme. The surface tension force is treated as a pressure gradient and an additional Poisson equation is solved. Transient and steady state bubble deformations, rise velocities and aspect ratios are presented.
\end{abstract}

Keywords: Gas-liquid flows; Bubble dynamics; VOF; confinement; square duct

\section{Introduction}

The dynamics of a single buoyant bubble in a stagnant liquid is a classical problem in fluid mechanics and has attracted the attention of numerous researchers for well over a century.

*pkumar8@illinois.edu

${ }^{\dagger}$ spvanka@illinois.edu 
Early works of Allen (1900), Hadamard (1911), Rybczynski (1911), Bond (1927) and Bond and Newton (1928) examined the validity of Stokes' drag law (of a rigid sphere) for a rising bubble and a falling liquid drop. Since bubbles and drops allow internal circulation and tangential velocity at their surfaces, Stokes' theory needed correction to account for the different boundary condition (Bond (1927), Bond and Newton (1928) and Levich (1962)). The motion and deformation/break-up of a bubble is influenced by several flow and fluid properties. While the rise velocity is mainly governed by the balance of buoyancy, drag and lift forces on the bubble, the shape of the bubble is influenced by the shear imposed by the surrounding liquid motion and the surface tension force acting at the interface. The various parameters governing the bubble rise and deformation can be grouped in two nondimensional numbers:

$$
\begin{aligned}
\text { Bond number }(B o) & \equiv \frac{\rho_{l} g d^{2}}{\sigma} \\
\text { Morton number }(M o) & \equiv \frac{g \mu_{l}^{4} \Delta \rho}{\rho_{l}^{2} \sigma^{3}}
\end{aligned}
$$

The Morton number is related solely to the fluid properties while the Bond number depends on the bubble diameter and surface tension. We can also define an Archimedes number as,

$$
A r \equiv \frac{\rho_{l}^{2} g d^{3}}{\mu_{l}^{2}}
$$

When $\Delta \rho=\left(\rho_{l}-\rho_{g}\right) \simeq \rho_{l}$, the Morton number can be shown to be equal to $B o^{3} / A r^{2}$.

Several early studies (Rosenberg (1950), Moore (1959) and Aybers and Tapucu (1969)) have documented the various shapes of the bubble over a wide range of Bond and Morton numbers. In low Morton number $\left(M o<1 \times 10^{-8}\right)$ liquids, the rise velocity first increased, then attained a maximum and decreased to reach a minimum value and then rose gradually with bubble diameter. The terminal shape ranged from spherical to oblate ellipsoidal to unstable skirted shapes. Smaller and larger bubbles rose in rectilinear and oscillatory paths respectively. In high Morton number $\left(M o>10^{-3}\right)$ liquids the rise velocity continuously increased with bubble diameter. The terminal shape also ranged from spherical to oblate ellipsoidal to skirted shapes. The temperature changes and impurities of the surrounding liquid also play an important role in the bubble dynamics (Aybers and Tapucu (1969)). The various experimental studies were combined in the form of charts by Grace (1973), Grace et al. (1976) and Bhaga and Weber (1981). The Grace chart is valid primarily for unconfined ducts. However, for confined ducts shape of the duct and the confinement ratio $\left(\equiv d / d_{\text {hyd }}\right)$, 
where $d_{h y d}$ is the hydraulic diameter of the duct, will be important.

Since the work of Bhaga and Weber (1981), a number of further experimental studies of unconfined bubbles have been conducted (e.g. Li and Schneider (1993), Ellingsen and Risso (2001), Shew et al. (2006), Sanada et al. (2007), Zhang et al. (2008), Legendre et al. (2012), and Maldonado et al. (2013)). Depending on the Bond and Morton numbers different bubble trajectories including rectilinear, zigzag, spiral and helical paths were observed. It was observed that during upward motion the bubble first rises in a rectilinear path for a small period of time, then the path transforms into a two-dimensional zigzag path and later evolves into an unstable spiral trajectory. Sanada et al. (2007) reported that no wake structures were observed for bubbles rising in rectilinear paths and but double-threaded vortices were present behind bubbles rising in axisymmetric helical paths. Ravelet et al. (2011) experimentally investigated deformation of a bubble rising in a uniform turbulent flow and explored the effects of sliding motion of bubble due to buoyancy.

A number of numerical studies of single and multiple bubbles rising in unconfined domains have used either Volume of Fluid (VOF) method (Hirt and Nichols (1981), DeBar (1974), Noh and Woodward (1976), Gueyffier et al. (1999), and Francois et al. (2006)), Level Set (LS) method (Osher and Sethian (1988) and Osher and Fedkiw (2003)), Coupled Level Set and Volume of Fluid (CLSVOF) method (Sussman and Puckett (2000), Sussman (2003) and Son (2003)) or the Front Tracking (FT) method (Unverdi and Tryggvason (1992) and Hua and Lou (2007)). In addition, several studies have used the mesoscopic Lattice Boltzmann method (LBM) (Inamuro et al. (2004), Amaya-Bower and Lee (2010), Cheng et al. (2010) and Amaya-Bower and Lee (2011)). Where applicable the numerical results were compared with the Bhaga and Weber (1981) and Grace (1973) charts. The effects of Reynolds number, Bond number, density ratio and viscosity ratio on bubble rise dynamics were studied (e.g. Chen et al. (1999), Ohta et al. (2005), Ohta et al. (2008), Hua and Lou (2007), Hua et al. (2008), Yu and Fan (2008), Chakraborty et al. (2013), Lalanne et al. (2013) and Tripathi et al. (2015a)). In addition, several researchers (e.g. Dandy and Leal (1989), Takagi et al. (1994), Tomiyama et al. (2002) and Yang et al. (2003)) have performed theoretical studies of the motion of a rising bubble.

Compared with the number of experimental and computational studies of bubble dynamics in unconfined domains, there are a limited number of studies in confined geometries. Studies of bubble motion in confined situations were reported by Krishna et al. (1999), FigueroaEspinoza et al. (2008), Roig et al. (2012), Böhm et al. (2014), Keshavarzi et al. (2014) and 
Tripathi et al. (2015b). Krishna et al. (1999) explored the effects of the wall on the rise velocity of a single gas bubble rising in a vertical liquid column. The bubble diameter $\left(d_{b}\right)$ varied between $3 \mathrm{~mm}$ and $80 \mathrm{~mm}$ and the tube diameter $\left(D_{T}\right)$ varied between $10 \mathrm{~mm}$ and $630 \mathrm{~mm}$. The rise velocity increased with increasing $D_{T} / d_{b}$ and became independent of $D_{T}$ for $D_{T} / d_{b}>8$. For the spherical cap bubbles $\left(B_{o}>40\right)$, the rise velocity obtained from experiments matched closely with the correlations. Figueroa-Espinoza et al. (2008) studied the effects of confinement on the motion of a single bubble in inertia dominated regime in vertical rectangular channels. For $R e<70$ and a range of confinement parameters, bubbles followed rectilinear paths. However, for $R e>70$ and all confinement ratios the bubbles followed zigzag paths.

Roig et al. (2012) studied the motion of a flattened bubble rising in a thin gap (confinement ratio less than one) between walls at high Reynolds number. This study was conducted over a wide range of Archimedes and Bond numbers. The Archimedes number varied between 10 and $10^{4}$ and Bond number varied between $6 \times 10^{-3}$ and 140 . The terminal Reynolds number varied as $A r / 2$ for a wide range of $A r$. Keshavarzi et al. (2014) considered bubble rise in a thin rectangular channel and their computations assumed two-dimensionality. Böhm et al. (2014) investigated the effects of channel depth, bubble size, superimposed liquid velocity and fluid rheology on the motion of a single bubble in a narrow rectangular channel. The bubble with an equivalent diameter larger than the channel gap rose in a two-dimensional zigzag path in a Newtonian fluid. However, in a non-Newtonian fluid, a bubble under similar conditions rose in rectilinear path. The bubble with equivalent diameter smaller than channel depth, due to lesser order of confinement in both directions, followed a helical path in a Newtonian fluid. Tripathi et al. (2015b) studied the effects of thermal gradient of surrounding liquid on bubble rise dynamics in self rewetting fluid (surface tension has parabolic dependence on temperature) and linear fluid (surface tension decreases linearly with temperature). They observed that at sufficiently small Bond number the motion of bubble can be arrested in self rewetting fluid.

Summarizing, bubble deformation and rise are very complex phenomena, governed by three important parameters: a) Bond or Eotvos number; b) Morton number and c) confinement ratio, besides the ratios of densities and viscosities. The unconfined bubble dynamics can be summarized by the Grace (1973) and Bhaga and Weber (1981) charts. However, not many studies have characterized bubble dynamics under confinement. In this work we report results of a numerical study of the effect of duct confinement of bubble dynamics at high and low Bond and Morton numbers using an accurate VOF based numerical method (Wang and 
Tong (2008)). The sharp surface force (SSF) method in conjunction with pressure boundary method is used for modeling of the surface tension forces and calculation of the pressure field due to surface tension. Section 2 describes the numerical scheme in detail. The code validation for the bubble rise in an infinite domain are presented in section 3 along with grid-independent studies. In section 4, we present results of simulations performed and draw conclusions on the effects of confinement ratio for different Bond and Morton numbers. A summary of the present results and the major findings are given in section 5 .

\section{$2 \quad$ Numerical Method}

\subsection{Governing equations}

We first assume the flow to be incompressible and write the mass continuity equation

$$
\frac{\partial \rho}{\partial t}+\nabla \cdot(\rho \mathbf{u})=0
$$

as a volumetric continuity equation

$$
\nabla \cdot \mathbf{u}=0
$$

The momentum equations are given by

$$
\frac{\partial(\rho \mathbf{u})}{\partial t}+\nabla \cdot(\rho \mathbf{u u})=-\nabla p+\nabla \cdot\left(\mu\left(\nabla u+\nabla^{T} u\right)\right)+\int_{\Gamma} \sigma_{f} \kappa_{f} \mathbf{n}_{f} \delta\left(\mathbf{x}-\mathbf{x}_{f}\right) d s+\rho \mathbf{g}
$$

In the above equations, $\mathbf{u}$ is fluid velocity, $p$ is pressure, $\rho$ is mixture density, $\mu$ is the dynamic viscosity, $\sigma$ is surface tension coefficient, $\Gamma$ is the interface, $\kappa_{f}$ is mean interface curvature, $\mathbf{n}_{f}$ is interface normal, $\delta$ is the delta function, $\mathbf{x}$ is the spatial location where equation is solved, $\mathbf{x}_{f}$ is the position of interface, $s$ is distance along the interface and $\mathbf{g}$ is acceleration due to gravity.

It is advantageous to write the momentum equation in non-dimensional form to classify and understand the flow in terms of non-dimensional variables. For the present bubble dynamics problem, the following non-dimensionlization can be used. The starred variables are defined as:

$$
\mathbf{x}^{*}=\frac{\mathbf{x}}{d} ; \quad \mathbf{u}^{*}=\frac{\mathbf{u}}{\sqrt{g d}} ; \quad t^{*}=\sqrt{\frac{g}{d}} t ; \quad \rho^{*}=\frac{\rho}{\rho_{l}} ; \quad \mu^{*}=\frac{\mu}{\mu_{l}} ; \quad p^{*}=\frac{p}{\rho_{l} g d} ; \quad \kappa^{*}=d \kappa ; \quad \mathbf{g}^{*}=\frac{\mathbf{g}}{g}
$$


where $d$ is bubble diameter, $\rho_{l}, \mu_{l}$ are liquid density and viscosity respectively. By dropping the * superscript from the non-dimensional momentum equations we can write them as,

$$
\frac{\partial(\rho \mathbf{u})}{\partial t}+\nabla \cdot(\rho \mathbf{u u})=-\nabla p+\frac{1}{A r^{1 / 2}} \nabla \cdot\left[\mu\left(\nabla \mathbf{u}+\nabla^{T} \mathbf{u}\right)\right]+\frac{1}{B o} \int_{\Gamma} \kappa_{f} \mathbf{n}_{f} \delta\left(\mathbf{x}-\mathbf{x}_{f}\right) d s+\rho \mathbf{g}
$$

\subsection{Solution procedure}

In the VOF method, an evolution equation for the liquid volume fraction $(\alpha)$ is solved. The evolution equation for the liquid volume fraction can be derived as,

$$
\frac{\partial \alpha}{\partial t}+\mathbf{u} \cdot \nabla \alpha=0
$$

The fluid density and viscosity are calculated as

$$
\begin{array}{r}
\rho=\alpha \rho_{l}+(1-\alpha) \rho_{g} \\
\mu=\alpha \mu_{l}+(1-\alpha) \mu_{g}
\end{array}
$$

Here $\rho_{l}, \rho_{g}$ are the liquid and gas densities, and $\mu_{l}, \mu_{g}$ are the liquid and gas viscosities. There are several approaches available in the literature for the solution of this VOF equation. We have used the geometry construction method (Rider and Kothe (1998)) with the normals to the interface calculated from liquid volume fraction $(\alpha)$ using second-order central differencing of the derivatives.

The second order operator split method of Noh and Woodward (1976), Li (1995), Ashgriz and Poo (1991) and Francois et al. (2006) is used for the solution of the VOF equation. In this method the advection along one direction (either $\mathrm{x}, \mathrm{y}$ or $\mathrm{z}$-direction) is done as a first step and then the advection along the other directions are done. The order of advection is cycled to remove any directionally-biased errors introduced due to splitting. First order explicit scheme is used for approximation of the time derivatives. Each step is discretized by the finite volume method, e.g. the x-direction step is written as

$$
\alpha^{*}=\alpha^{n}-\frac{\left(\alpha^{n} u^{n} \Delta t d \mathcal{A}\right)_{\left(i+\frac{1}{2}, j, k\right)}-\left(\alpha^{n} u^{n} \Delta t d \mathcal{A}\right)_{\left(i-\frac{1}{2}, j, k\right)}}{d \mathcal{V}}+\left(\alpha_{(i, j, k)}^{n} \frac{u_{\left(i+\frac{1}{2}, j, k\right)}^{n}-u_{\left(i-\frac{1}{2}, j, k\right)}^{n}}{\Delta x}\right) \Delta t
$$


where $d \mathcal{V}$ is the cell volume. The terms $\left(\alpha^{n} u^{n} \Delta t d \mathcal{A}\right)_{\left(i+\frac{1}{2}, j, k\right)}$ and $\left(\alpha^{n} u^{n} \Delta t d \mathcal{A}\right)_{\left(i-\frac{1}{2}, j, k\right)}$ are the liquid volumes leaving and entering through the right and the left faces. These volumes are computed geometrically using the interface description. The mass fluxes through cell faces are calculated from the volumes of individual phases crossing the cell faces and their densities. This method is known to be accurate and robust to handle large density ratios (Rider and Kothe (1998)).

The proper inclusion of the surface tension force is a very important issue in reducing the spurious velocities. The surface tension force is a force that acts at the interface. In a static situation the pressure difference across the interface must balance the surface tension force. For representing the surface tension, we use the Sharp Surface Force (SSF) (Francois et al. (2006) and Wang and Tong (2008)). The concept of SSF addresses the pressure jump across the interface directly. In this method, the surface tension term in the Navier-Stokes equations is written as a pressure gradient term. This pressure gradient exactly balances the surface tension force generated due to the presence of the interface. Thus,

$$
\frac{1}{B o} \int_{\Gamma} \kappa_{f} \mathbf{n}_{f} \delta\left(\mathbf{x}-\mathbf{x}_{f}\right) d s=-\nabla \tilde{p}
$$

where $\tilde{p}$ is a pressure field solely generated due to the surface tension force at the interface. Using the continuity equation and the assumption that this pressure gradient does not generate any velocity, we can derive a Poisson equation for $\tilde{p}$ as

$$
\nabla \cdot\left(\frac{\nabla \tilde{p}}{\rho}\right)=0
$$

The gradients at the cell faces are evaluated with the knowledge of the sudden jump at any interface, giving

$$
\nabla \cdot\left(\frac{\nabla \tilde{p}}{\rho}\right)=F^{x+}-F^{x-}+F^{y+}-F^{y-}+F^{z+}-F^{z-}
$$

where $F^{x+}$ is defined as,

$$
F^{x+}=-\left(\frac{\sigma \kappa}{\rho \Delta x^{2}}\right)_{\left(i+\frac{1}{2}, j, k\right)}
$$

Similar expressions can be written for $F^{x-}, F^{y+}, F^{y-}, F^{z+}$ and $F^{z-}$. These forces are the source terms in the pressure equation. When there is no interface present between two cells the force on the corresponding face is zero, otherwise it is computed from the above 
expressions such as eq. (15). The above equation is further improved by considering exact geometrical position of the pressure jump.

The Poisson equation for $\tilde{p}$ is solved by a multigrid accelerated red black SOR relaxation scheme. The pressure gradient $-\nabla \tilde{p}$ is then added to the discrete momentum equations in place of the surface tension term. We have ensured that the method reduces the spurious velocities to machine zero for a static bubble with exact analytical curvature prescribed. However, when the curvature is numerically computed small spurious velocities, much smaller than flow velocities, are generated. In this work we have used the height function method (Rudman (1998) and Cummins et al. (2005)) for curvature computations.

The momentum and continuity equations are discretized on a collocated Cartesian grid using a finite volume method. The terms in the non-dimensional momentum equation given by (eq. (7)) are integrated with second-order accuracy in time and space using AdamsBashforth time advancement. The convection term $(\nabla \cdot \rho \mathbf{u u})$ is computed geometrically with linear interpolation for the face velocity. The pressure gradient term is written at cell faces and in the form of $\frac{\nabla p}{\rho}$. Coupling the pressure gradient and density together eliminates any ambiguity in density interpolation and leads to a robust method. The viscous term is computed by second order central differencing. Linear interpolation is used for face densities and viscosities.

\subsection{Boundary conditions}

All six boundaries of the square duct are walls. Therefore no-slip and no-penetration boundary conditions are applied at these walls.

$$
\begin{aligned}
& u_{\text {wall }}=v_{\text {wall }}=w_{\text {wall }}=0 \\
& \left(\frac{\partial p}{\partial \hat{n}}\right)_{\text {wall }}=\left(\frac{\partial \tilde{p}}{\partial \hat{n}}\right)_{\text {wall }}=0
\end{aligned}
$$

where $\hat{n}$ is the wall normal. 


\section{Implementation, validation and study of grid indepen- dency}

The equations presented in Section 2 are solved with an in-house code, CUFLOW. CUFLOW is a general purpose code for simulating laminar and turbulent flows in complex domains. The code employs Cartesian grids to integrate the three-dimensional unsteady incompressible Navier-Stokes equations. The continuity and momentum equations are solved using a fractional step method. The CUFLOW has been programmed to run entirely on multiple graphics processing units (GPU). Details and validations of CUFLOW are given in the works of Vanka et al. (2011), Chaudhary (2011) and Shinn (2011). The multi-GPU code has been benchmarked for a three-dimensional lid-driven cavity problem with a grid of $128 \times 128 \times 512$ control volumes. The algorithm on a single GPU (NVIDIA Tesla K20x) is approximately 42 times faster than that on a single CPU core (AMD $62762.3 \mathrm{GHz}, 16$ cores). The algorithm on four GPUs is approximately 20 times faster than that with four cores of the CPU. The scaling between single and four GPUs is approximately 3.13. The VOF algorithm was validated for a static bubble with the gravity field switched off. The 'spurious' velocities produced in this case were of the order of $10^{-5}$. These can be solely attributed to the approximations caused by the height function method.

We next validated the accuracy of numerical method with some of the results from works of Grace (1973) and Bhaga and Weber (1981). In Fig. 1, the second column shows the experimental test condition. In this experiment, the Bond and Morton numbers were prescribed by fixing the fluid properties and the bubble size. We have followed the same procedure in our numerical computations. These experiments were performed in a circular tube with very large confinement ratio $\left(D_{\text {tube }} / D_{\text {bubble }}\right)$. In our calculations, we have prescribed a confinement ratio of 8 . The density and viscosity ratios for these calculations were approximately 1000 and 100. We compare terminal shape and terminal velocity for two separate cases: A) spherical bubble $(B o=17.7$ and $M o=711)$; and B) oblate ellipsoidal cap $(B o=243$ and $M o=266$ ). For both of these cases Bhaga and Weber (1981) reported the terminal Reynolds number and terminal shape.

From Fig. 1, it can be seen that the predicted terminal shapes are similar to experimentally observed shapes. However, in case A the experimental shape is ellipsoidal. We believe the deformation in the experiments may be a result of changes in surface tension due to impurities in the liquid or other effects. This difference was seen also in computations of air- 
water flows (Jin et al. (submitted)) unless the experiments were done with ultra-pure water and filtered air such as by Wu and Gharib (2002). For both cases the predicted terminal Reynolds numbers are slightly underestimated. The difference between experimental and numerical results is less than $2.5 \%$.

We next performed grid refinement studies on the motion of a bubble rising in a stagnant liquid pool. In this case we selected Bond and Morton numbers to be 243 and 266 respectively, and the confinement ratio to be 4. Four set of grids, with 32, 48, 64 and 96 control volumes per bubble diameter were chosen for this study. Figure 2(a) shows the transient bubble rise velocities for the four grids. The inset in fig. 2(a) shows the zoomed-in view of rise velocities. It can be observed that the rise velocities show grid convergence and the relative error in velocity between 32 and 64 control volumes per bubble diameter are $2.0 \%$ compared to $0.24 \%$ relative error between results with 64 and 96 control volumes per bubble diameter.

Figure 2(b) shows the terminal shapes of the bubble for different grids. We can observe from the figure that the terminal shapes of the bubble are very close for all grids. The inset figure shows the region of maximum gradient in the deformed shape of the bubble. Paying close attention to the inset figure, we can deduce that the result with 64 control volumes per bubble diameter is very close to the finest grid result. Therefore 64 control volumes per bubble diameter are used for all computations in this study.

\section{Results}

We now present results of a systematic study of the effects of confinement ratio on bubble deformation, transient aspect ratio and transient rise velocity in a square duct. Three confinement ratios, four Bond numbers and two Morton numbers have been simulated. Figure 3 shows the geometry used for this study with gravity acting downwards. The air bubble is initially placed at a distance of two diameters above the bottom boundary in the center of the cross-section.

The motion of the bubble is dictated by the external forces acting on it. Several competing forces act on a bubble as it moves through the liquid: i) an upward buoyant force due to density difference; ii) a viscous force on the bubble surface restraining its motion; and iii) a surface tension force acting to reduce bubble deformation. The balance of these forces leads to a steady bubble shape and a terminal velocity. The effects of these forces can be studied 
systematically through relevant non-dimensional parameters.

In the present study, the width of the duct is prescribed by the confinement ratio. The height is taken as 16 bubble diameters in all cases. Based on the grid refinement study, we have used 64 control volumes per bubble diameter. We consider two Morton numbers namely 0.001 and 0.01. For each of these values we study four Bond numbers namely 1, 10, 50 and 100. Depending on the Bond number the deformed bubble shapes vary from spherical to oblate spheroidal to spherical cap to a flattened disk-like structure. The corresponding fluid properties are listed in table 1. The Bond number 1, 10, 50 and 100 are represented by bubbles of $2.309 \mathrm{~mm}, 7.302 \mathrm{~mm}, 16.33 \mathrm{~mm}$ and $23.09 \mathrm{~mm}$ diameters respectively.

The rise velocity is calculated as a volumetric average of the vertical velocity component along the interface $(\alpha=0.5)$ using the expression

$$
w_{t}=\frac{\int_{\alpha=0.5} \alpha w d v}{\int_{\alpha=0.5} \alpha d v}
$$

The aspect ratio of the bubble is calculated in the central yz plane (shown in Fig. 4) using the expression

$$
\text { Aspect ratio }=\frac{\text { width }}{\text { height }}
$$

\subsection{Morton number $=0.001$}

We first present the results of lower Morton number. A lower Morton number implies lower liquid viscosity. Due to the hydrostatic pressure difference between the top and bottom surfaces of the bubble there is a net upward force on it. This imbalance in force leads to an upward motion of the bubble and subsequent motion in the surrounding liquid. The drag force on the bubble is smaller hence the bubble moves faster through the liquid and deforms by larger amount. Further, as the confinement ratio increases, the reduction in wall shear makes the rise velocities higher.

Figure 5 shows a typical three-dimensional perspective of the bubble deformation for $B o=$ 1, 10,50 and 100 and $M o=0.001$ at various non-dimensional times. The bubble is rising vertically, but for ease of presentation we show the three-dimensional surfaces in a horizontal

arrangement. The simulation time increases from left to right and the Bond number increases from top to bottom. It can be seen from Fig. 5 that for Bond number of 1 , the bubble remains 
nearly of the same spherical shape as the bubble moves upward (shown left to right here). At higher Bond numbers the bubble deforms considerably. At $B o=10$ the bubble quickly deforms into an oblate spheroidal shape and rises with that shape. Both for $B o=50$ and $B o=100$ the bubble deforms into a spherical cap and goes through shape oscillations to reach a disk-like structure. Comparing these two Bond numbers we can also notice that both bubbles go through similar transients, however, these transients are observed at earlier times for $B o=50$. It can also be seen that a number of very small gas particles break away from the bubble for Bond number of 100. This break up may be due to insufficient resolution at the tips, but the cumulative volume of all the satellite bubbles is less than $2 \%$ of the total gas volume, hence the dynamics of the bulk are unaffected. The same three-dimensional shapes are seen at other confinement ratios (hence are not shown).

Figure 6 shows two-dimensional cross-sectional shapes on the center plane and their positions at different times for different confinement ratios. We observe that for all the three confinement ratios and all four Bond numbers the bubble rose in a straight line. The shape and rise path seen here are consistent with observations of Moore (1959) for the rise of a bubble in an unconfined medium and of Figueroa-Espinoza et al. (2008) in a confined duct. The bubbles traveled a smaller distance at smaller confinement ratios than in ducts with higher confinement ratios. For $B o=1$, in 10 time units the bubble rose by approximately 2, 3 and 4 diameter units in the duct with confinement ratios of 2,3 and 4 . The distance traveled by the bubble increases with increasing Bond number which can be seen from figures 6(b), 6(c) and 6(d). For highest Bond number $(B o=100)$ the bubble traveled by 5.17, 5.93 and 6.25 diameters respectively.

Fig. 6 also shows the deformation history of the bubbles at different Bond numbers. For $B o=1$, the bubble does not deform much. For $B o=10$ the bubble deforms into an ellipsoidal shape and remains ellipsoidal for the rest of its ascent. Further increase in Bond number results in a complex deformation pattern where the bottom surface of the bubble goes through oscillations and top surface remains nearly spherical. Both for $B o=50$ and $B o=100$ the bubble deforms significantly. At these Bond numbers the bubble transforms from the starting spherical shape into different shapes from hemispherical to a disk-like structures. During the initial acceleration stage the inertial force dominates the surface tension force, therefore larger deformation is observed. It is seen in Fig. 6(c) and Fig. 6(d) that the spherical bubble transforms into a spherical cap at $t=2.0$ for $B o=50$ (and $t=2.5$ for $B o=100)$. This qualitative characteristic is common for all three confinement ratios, but the extent of deformation is different at low values of the duct width. Once the bubble 
has deformed to its maximum extent (which happens approximately at $t=2.0$ for $B o=50$ and $t=2.5$ for $B o=100$ ), surface tension force starts to dominate. This leads to retraction of the bottom surface. The retraction process takes place between $t=2.0$ and $t=3.5$ for $B o=50$ (and between $t=2.5$ and $t=5.0$ for $B o=100$ ). In the case of $B o=50$, the bottom surface of the bubble at $t=3.5$ is nearly flat for $C R=2$ but for higher confinement ratios the bottom shapes are deformed. After this, the relaxation of the bottom surface ceases and a transition towards steady state happens. A similar process of relaxation of bottom surface takes place in the case of $B o=100$ as well. It is worth mentioning that in the steady state the bubble shapes are flatter for $C R=3$ and $C R=4$ than for $C R=2$ both for $B o=50$ and $B o=100$ cases.

The transient rise velocity of the bubble is appreciably influenced by the confinement ratio, and is shown in Fig. 7. For all $C R$ and $B o$ the rise velocity increases with time to reach the steady state. However, the rise velocity and time taken to reach a steady state depends on the confinement ratio and the Bond number. The rise velocity is estimated by surface integration of the z-velocity along the bubble interface. No significant oscillation in rise velocity is observed at $B o=1$ for all the confinement ratios. However, as the Bond number is increased, oscillation in rise velocity is observed. For $B o=10$ an initial oscillation is observed, with the magnitude largest for $C R=2$ and decreasing for confinement ratios of 3 and 4. The terminal rise velocities are $0.612,0.678$ and 0.731 respectively, for $C R=2,3$ and 4. The oscillations in rise velocities increase for $B o=50$ and $B o=100$. This trend can be seen in figures $7(\mathrm{c})$ and $7(\mathrm{~d})$. For $B o=50$ the rise velocity goes through two peaks with its first maximum at $t=0.6$ where the rise velocities are $0.653,0.696$ and 0.709 respectively for the different confinements. The effect of confinement is again to decrease the rise velocities, as a result of the shear from the walls. The rise velocity gradually decreases to reach a quasi-steady state at $t=4.0$. The terminal velocities for $B o=50$ are 0.526, 0.609 and 0.661 for confinement ratios of 2, 3 and 4. A similar behavior is seen for $B o=100$ except the velocities reach steady state at $t=4.5$ with corresponding values of $0.53,0.62$ and 0.66 for the three confinement ratios. For $B o=100$, the second peak of rise velocity is higher than the first peak indicating a larger oscillation.

We next study the aspect ratio of the bubble for the three confinement ratios. Figure 8 shows the transient aspect ratios for four Bond numbers. The transient aspect ratio sheds further light on the nature of transient deformation of the bubble. The aspect ratio of the bubble is calculated as the ratio between the maximum width of the bubble to the minimum distance between the top and bottom surfaces of the bubble. As the bubble rises, it deforms, and 
once all the forces acting on the bubble come to a balance it assumes a steady shape. Thus the aspect ratio first increases with time and then reaches a steady state. We observe that the aspect ratio increases with confinement ratio. The aspect ratio also increases with Bond number with a larger effect of the confinement ratio. The aspect ratios for all confinements are close to 1 for Bond number of 1 , but at higher Bond numbers the aspect ratio increases. For Bond number of 10 the steady state aspect ratios of the deformed bubble are 1.68, 1.88 and 1.96 respectively for $C R=2,3$ and 4 . These numbers indicate an ellipsoidal steady shape for all confinements.

For higher Bond numbers, there are significant oscillations in the aspect ratio. These oscillations are seen to be a result of the oscillations of the bottom surface of the bubble. In the initial acceleration stage the bottom surface deforms significantly to form a spherical cap structure which leads to high values of the aspect ratios. In the case of $B o=50$ the oscillations in the aspect ratio continue for longer periods and a steady state is not observed in the time period computed in this study. The oscillations however decrease with time. The oscillations are relatively small at $t=9.5$ with, the aspect ratios being $2.56,3.26$ and 3.55 respectively.

In the case of $B o=100$ however, only one peak with aspect ratio as high as 27 is observed. The diameter of the bubble in the case of $B o=100$ is approximately $37 \%$ higher than that in the case of $B O=50$. Hence the initial hydrostatic pressure difference between the top and bottom surfaces of the bubble is higher than that of $B o=50$. The higher bubble diameter also results in a higher Archimedes number therefore the viscous forces are smaller. The bubble thereby deforms more to a thin neck resulting in a large aspect ratio. This is observed at $t=1.7$ with the corresponding aspect ratios being $9.40,21.50$ and 27.33 respectively for the three confinement ratios. As the confinement ratio decreases, the deformation decreases, giving a smaller aspect ratio. After the maximum deformation the surface tension forces become prominent and the bottom surface of the bubble retracts to a stable and lesser deformed state at $t=6$ with the aspect ratios as 2.90, 4.32 and 4.93 for the three confinement ratios respectively.

Figure 9 shows the bubble shape at $t=9,9.5$ and 10 on the central $y z$ plane for $B o=10$ and 50. The three bubble shapes are overlaid by shifting their top surface to align with each other. We observe that in the case of $B o=10$ the shapes are identical at these times. In the case of $B o=50$ the top surfaces of the bubbles match identically. However the bottom surface at $t=9$ is different from that at $t=9.5$ and 10 . This trend is also seen in the Figures 
8(b) and 8(c) where the bubble reaches steady state in the case of $B o=10$ and continues to oscillate in the case of $B o=50$. Hence the aspect ratio plots can be proxies for the bubble shape reaching a steady state.

In figure 10 we show the streamlines for $B o=100$ and $M o=0.001$ at a representative time of $t=10$. The streamlines are plotted in a stationary frame of reference of the bubble. We see that as the confinement ratio decreases, because the distance between the wall and the bubble surface also decreases, the surrounding liquid has lesser space between the wall and the bubble surface. Thus for confinement ratio of 2 the streamlines are squeezed between the wall and the bubble surface. For higher confinement ratios the streamlines are however, farther spaced. As the distance between the streamlines decreases, the $z$-velocity gradient in the wall normal direction increases leading to a higher drag on the bubble. The drag on the bubble decreases with an increase in the confinement ratio. This is reflected in the figure 7 (b) as rise velocity increases with an increase in the confinement ratio.

Figure 12 shows the top views of the $z$-vorticity iso-surfaces at $t=10$ for all three confinement ratios and $B o=100$ and $M o=0.001$. Two values of clockwise and anti-clockwise vorticities $\left(\omega_{z}= \pm 0.05\right.$ and \pm 0.5$)$ have been plotted. The maximum and minimum values of $\omega_{z}$ are approximately 10 and -10 respectively. However, these values are localized near the bubble interface, therefore we have plotted smaller values of the vorticity. It can be seen that the confinement ratio plays a significant role in the distribution of the vorticities. The vortical structures of $\omega_{z}= \pm 0.05$ originating from the bubble are connected to the wall in the case of $C R=2$ as seen from Fig. 11(a). As the confinement ratio increases the connectivity vanishes. The vortical structures of $\omega_{z}= \pm 0.5$ are mostly localized near the bubble interface and their size is dependent on the confinement ratio.

Figure 13 shows the instantaneous streamlines in $y z$ plane in the reference frame of bubble for the four Bond numbers, $C R=4$ and $M o=0.001$. It can be seen that the streamlines are symmetric about a vertical line passing through the bubble centroid for all cases. In the case of $B o=1$ the streamlines are symmetric about a horizontal line passing through bubble centroid which are similar to those observed for flow past a solid sphere at low Reynolds number. In the case of $B o=10$ the streamlines are not symmetric about a horizontal line and no standing wake behind the bubble is observed. For higher Bond numbers, the bottom portion of the bubble deforms and leads to the creation of recirculation zones. Both of these recirculation zones are attached to the bubble, however they do not penetrate the bubble. It can be also noticed that there are small recirculation zones inside the deformed bubbles. 
The recirculation zones behind the bubbles are bigger for $B o=100$ than for $B o=50$.

\subsection{Morton number $=0.01$}

Increase in fluid viscosity by approximately a factor of 2 increases the Morton number by 10 (fourth power). Increase in fluid viscosity will reduce the bubble deformation through smaller fluid velocities. In this section, we present results for $M o=0.01$. Because the qualitative effects of confinement are similar to those at lower $M o$, we present these results and discuss them only briefly. The plots shown are similar to figures 5 to 8 . Figure 14 shows the three-dimensional perspective of the bubble deformation for $B o=1,10,50$ and 100 and $M o=0.01$ at various times. The bubble dynamics at this Morton number are similar to observations made in the case of $M o=0.001$. However, the deformations are smaller at the higher Morton number. This is more clear from results at $B o=100$. For low Morton number (Fig. 5) several tiny satellite bubbles formed due to insufficient local resolution and low viscosity. No such pattern can be seen in Fig. 14. The rest of the features are similar at both Morton numbers and all Bond numbers.

Figure 15 shows the transient shapes and positions of the bubbles on the center plane for the three confinement ratios and four Bond numbers. It can be seen that the bubbles again rise in a straight line for all Bond numbers and confinement ratios. The bubble goes through a range of deformations from spherical, ellipsoidal, spherical cap and disk-like structures. The shapes at $M o=0.01$ are similar to those of $M o=0.001$ but as expected, quantitative differences are seen.

For $B o=1$, it can be observed from Fig. 15(a) that bubble remains spherical. In 10 time units the bubble rose by 1.31, 1.96 and 2.31 diameter units respectively, for confinement ratios of 2, 3 and 4. By comparing results between figures 6(a) and 15(a), we can say that the as the Morton number of flow (viscosity of surrounding liquid) increases the distance traveled by bubble decreases. For $B o=10$ (Fig. 15(b)), the terminal shapes of the bubble varied from asymmetric for $C R=2$ to symmetric ellipsoidal bubble for $C R=4$. In the case of $C R=2$ it can be seen that in the steady state the bubble is asymmetric with respect to its major axis. The bottom surface is flatter than the top surface. It can be seen that the distance between the top surface and the major axis is more than the distance between the bottom surface and the major axis. The differences in the top and the bottom surfaces of the ellipsoid decrease with confinement ratio and for the $C R=4$ case the symmetry along 
the major axis is almost restored. Similar to observations made in Fig. 6(b), the distance traveled by the bubble increases with increasing confinement ratio. The bubble rose by 4.26 , 5.55 and 6.00 diameter in 10 time units respectively in ducts with confinement ratios of 2,3 and 4 .

At larger Bond numbers the bubble deforms to a spherical cap at intermediate times and relaxes to a disk-like structure in the steady state. The trends are similar for both $B o=50$ and $B o=100$ at all confinement ratios. In the steady state a significant difference in the thickness of the middle portion of the bubble can be observed between $C R=2$ and $C R=4$. Terminal shape of the bubble for $C R=4$ is flatter and wider than that for $C R=2$. However, no such observations can be made between $C R=3$ and $C R=4$ at this stage. For $B o=50$ (Fig. 15(c)), in 10 time units the bubble rises by 4.84, 5.54, and 5.89 diameters units respectively, for confinement ratios of 2, 3 and 4. For $B o=100$ (Fig. 15(d)), in the steady state the bubble shape is hemispherical for a confinement ratio of 2 . But for the confinement ratios of 3 and 4 the steady shapes are moon shape with a flatter bottom surface. In this case in 10 time units the bubble rises by 5.04, 5.90 and 6.26 diameter units respectively, for confinement ratios of 2,3 and 4 .

Figure 16 shows the transient rise velocities for the three confinement ratios, four Bond numbers and $M o=0.01$. In the case of $B o=1$ there are small oscillations in the rise velocities for all confinement ratios. These oscillations are less than $2 \%$ of the terminal velocity and are likely due to the volumetric averaging procedure used on the Cartesian grid. For $B o=1$ the terminal velocities of the bubbles are $0.13,0.20$ and 0.23 respectively, for confinement ratios of 2,3 and 4. The terminal velocities of the bubbles for $B o=10$ are 0.44 , 0.58 and 0.63 respectively and these are shown in Fig. 16(b). For the cases of $B o=10$ and $C R=2$ and 3 initial overshoots in rise velocity are observed.

Rise velocities for $B o=50$ and $B o=100$ are shown in Fig. 16(c) and Fig. 16(d). It can be seen that oscillations in the rise velocities at higher Bond numbers are far more significant than that at lower Bond numbers. These oscillations are related to the oscillations of the bottom surface of the bubble during its ascent. As reported in the section 4.1 the deformation and oscillation of the bubble are considerably larger at higher Bond numbers. From rise velocities of $B o=50$ and $B o=100$ we can deduce that bottom surface goes through two oscillations before it reaches a steady state. In the steady state the rise velocities are 0.506, 0.603 and 0.644 respectively for $B o=50$. In the case of $B o=100$ the terminal rise velocities are $0.512,0.607$ and 0.654 respectively, for confinement ratios of 2,3 and 4 . It can be deduced 
from these results that the rise velocities are comparable for both $B o=50$ and $B o=100$.

Figure 17 shows the transient aspect ratios for all confinement ratios, all Bond numbers and $M o=0.01$. Similar to results presented in section 4.1 the aspect ratio increases with increasing Bond number and confinement ratio. For $B o=1$ the aspect ratios are close to 1. For $B o=10$ (Fig. 17(b)) the aspect ratios in the steady state are 1.28, 1.51 and 1.57 respectively. For $B o=50$ and $B o=100$ the bottom surface of the bubble goes through oscillations which is reflected as the aspect ratio oscillations. The aspect ratio for $B o=50$ reaches a steady state at $t=6.5$ with aspect ratios of $2.36,3.12$ and 3.37 . For $M o=0.001$ (Fig. 8(c)) only a quasi-steady state was achieved. This indicates the higher viscous damping at the higher Morton number. For $B o=100$ (Fig. 8(d)) the aspect ratio reaches a quasi steady state at $t=6.50$ with the aspect ratios being $2.65,3.75$ and 4.12 respectively. It is also interesting to mention that the highest aspect ratio for $M o=0.001$ was approximately 27 whereas the highest aspect ratio for $M o=0.01$ is approximately 7.6.

From the above results, we can make a few interesting observations. We see that at low Bond numbers and both Morton numbers, there are no oscillations in the rise velocity as well as in the aspect ratio. This is because the viscous time scale $\left(\approx \sqrt[1 / 4]{B o^{3} / M o}=A r^{1 / 2}\right)$ is small, so that the buoyancy forces are equilibrated quickly with viscous effects. The time to reach a steady bubble aspect ratio by when the surface tension effects are also equilibrated with other forces is shorter at low Bond numbers. For high Bond number cases, the significant deformation and oscillatory rise velocity are due to both larger viscous and larger surface tension time scales which are a function of the Bond number. Hence for Bond numbers, buoyancy acts for a longer time deforming the bubble. The effect of confinement is to increase the wall shear stress to the surrounding flow and thus decrease the bubble velocity and deformation.

Table 2 summarizes the terminal Reynolds number for all the cases. It can be seen that the terminal Reynolds number increases with Bond number and confinement ratio but decreases with Morton number. The increase in terminal Reynolds number with Bond number is attributed to the higher bubble velocity due to the larger buoyancy force for larger bubbles. The viscosity is higher for higher Morton number cases, hence the drag force on the bubble will be larger and subsequently the terminal Reynolds number will be smaller. Similarly effects of wall are higher for low confinement ratio cases, therefore with increase in confinement ratio terminal rise velocity increases. It is also worthwhile mentioning that the increase in terminal Reynolds number is not linear with the confinement ratio. 


\section{Summary}

A multi-GPU based numerical method has been developed for the solution of the variable density and variable viscosity Navier-Stokes equations on a uniform collocated Cartesian grid. The volume of fluid method coupled with a three-dimensional geometry construction method is used for accurate representation and tracking of the interface. The geometry construction method is also used for calculation of the convection term. The pressure balance method which involves the coupling of pressure gradient and face density is used for treatment of the pressure gradient term and other body forces in the Navier-Stokes equations. An additional pressure Poisson equation is solved for inclusion of the surface tension force. The current implementation is stable at high density ratios $(\sim 1000)$ and generates only very small spurious velocities.

In this paper, we have characterized the effects of the three-dimensional duct confinement on bubble rise dynamics in a viscous liquid. We have explored the effects of confinement ratios (duct size/bubble diameter) on the transient bubble shapes, deformed bubble's aspect ratio and transient rise velocities. We also report the terminal bubble shapes and the terminal Reynolds number. Two Morton numbers, three confinement ratios and four Bond numbers have been selected. The results have been presented for two Morton numbers. For each Morton number four Bond numbers are considered.

It is seen that with the increasing Bond number from 1 to 100 the terminal bubble shape changes from spherical to oblate spheroidal to spherical cap to flatter disk-like structures. The transient aspect ratio decreases with increase in Morton number. For Bond number of 1, the bubble does not deform and corresponding transient aspect ratios are independent of the Morton number and confinement ratio. For higher Bond numbers, the deformation is significant and the transient aspect ratio increases with increase in Bond number and confinement ratio but decreases with increase in Morton number. Significant oscillations in the aspect ratio are also observed for high Bond numbers cases. However, these oscillations decreased with increase in Morton number.

The transient rise velocity is also dependent on the non-dimensional parameters. For Bond number of 1 the rise velocity was independent of the Morton number and confinement ratio. However, for higher Bond numbers the rise velocity increased with increase in Bond number and confinement ratio and decreased with increase in Morton number. It is also important to note that the increase in rise velocity is not linear with confinement ratio. The change 
in rise velocity between confinement ratios of 2 and 3 is larger than the change between confinement ratios of 3 and 4 . Significant oscillations in the rise velocity are present at higher Bond numbers but they decrease with increase in Morton number.

\section{Acknowledgments}

The authors thank the financial support from the Air Conditioning and Refrigeration Center (ACRC), University of Illinois at Urbana-Champaign, USA. This research is also part of the Blue Waters sustained-petascale computing project, which is supported by the National Science Foundation (awards OCI-0725070 and ACI-1238993) and the state of Illinois. Blue Waters is a joint effort of the University of Illinois at Urbana-Champaign and its National Center for Supercomputing Applications. The authors further thank NVIDIA Hardware Grant Program for providing the GPUs for an in-house workstation.

\section{References}

Allen, H. S., 1900. The motion of a sphere in a viscous fluid. Philosophical Magazine Series 550 (304), 323-338.

Amaya-Bower, L., Lee, T., 2010. Single bubble rising dynamics for moderate reynolds number using lattice boltzmann method. Computers \& Fluids 39 (7), 1191-1207.

Amaya-Bower, L., Lee, T., 2011. Numerical simulation of single bubble rising in vertical and inclined square channel using lattice boltzmann method. Chemical Engineering Science $66(5), 935-952$.

Ashgriz, N., Poo, J., Apr. 1991. FLAIR: flux line-segment model for advection and interface reconstruction. Journal of Computational Physics 93 (2), 449-468.

Aybers, N., Tapucu, A., 1969. The motion of gas bubbles rising through stagnant liquid. Wärme- und Stoffübertragung 2 (2), 118-128.

Bhaga, D., Weber, M., 1981. Bubbles in viscous liquids: shapes, wakes and velocities. Journal of Fluid Mechanics 105, 61-85. 
Böhm, L., Kurita, T., Kimura, K., Kraume, M., Oct. 2014. Rising behaviour of single bubbles in narrow rectangular channels in Newtonian and non-Newtonian liquids. International Journal of Multiphase Flow 65, 11-23.

Bond, W., 1927. Bubbles and drops and Stokes' law. Philosophical Magazine Series 74 (24), 889-898.

Bond, W., Newton, D. A., 1928. Bubbles, drops, and stokes' law. (paper 2). Philosophical Magazine Series 75 (30), 794-800.

Chakraborty, I., Biswas, G., Ghoshdastidar, P., Mar. 2013. A coupled level-set and volumeof-fluid method for the buoyant rise of gas bubbles in liquids. International Journal of Heat and Mass Transfer 58 (1-2), 240-259.

Chaudhary, R., 2011. Studies of turbulent flows in continuous casting of steel with and without magnetic field. Ph.D. thesis, University of Illinois at Urbana-Champaign.

Chen, L., Garimella, S. V., Reizes, J. A., Leonardi, E., 1999. The development of a bubble rising in a viscous liquid. Journal of Fluid Mechanics 387 (1999), 61-96.

Cheng, M., Hua, J., Lou, J., 2010. Simulation of bubble-bubble interaction using a lattice boltzmann method. Computers \& Fluids 39 (2), 260 - 270.

Cummins, S. J., Francois, M. M., Kothe, D. B., 2005. Estimating curvature from volume fractions. Computers \& Structures 83 (6-7), $425-434$.

Dandy, D. S., Leal, L. G., 11 1989. Buoyancy-driven motion of a deformable drop through a quiescent liquid at intermediate reynolds numbers. Journal of Fluid Mechanics 208, 161-192.

DeBar, R., 1974. Fundamentals of the KRAKEN code. Lawrence Livermore Laboratory, UCIR-760 (March).

Ellingsen, K., Risso, F., 2001. On the rise of an ellipsoidal bubble in water: oscillatory paths and liquid-induced velocity. Journal of Fluid Mechanics 440, 235-268.

Figueroa-Espinoza, B., Zenit, R., Legendre, D., Nov. 2008. The effect of confinement on the motion of a single clean bubble. Journal of Fluid Mechanics 616, 419-443. 
Francois, M. M., Cummins, S. J., Dendy, E. D., Kothe, D. B., Sicilian, J. M., Williams, M. W., Mar. 2006. A balanced-force algorithm for continuous and sharp interfacial surface tension models within a volume tracking framework. Journal of Computational Physics 213 (1), 141-173.

Grace, J., 1973. Shapes and velocities of bubbles rising in infinite liquids. Trans. Inst. Chem. Eng. 51 (2), 116-120.

Grace, J., Wairegi, T., Nguyen, T., 1976. Shapes and velocities of single drops and bubbles moving freely through immiscible liquids. Chemical Engineering Research and Design 54a, $167-173$.

Gueyffier, D., Li, J., Nadim, A., Scardovelli, R., Zaleski, S., Jul. 1999. Volume-of-Fluid interface tracking with smoothed surface stress methods for three-dimensional flows. Journal of Computational Physics 152 (2), 423-456.

Hadamard, J., 1911. Mouvement permanent lent d'une sphere liquide et visqueuse dans une liquide visqueux. Comptes rendus de l'Académie des sciences 152, 1735.

Hirt, C. W., Nichols, B. D., Jan. 1981. Volume of Fluid (VOF) method for the dynamics of free boundaries. Journal of Computational Physics 39 (1), 201-225.

Hua, J., Lou, J., Mar. 2007. Numerical simulation of bubble rising in viscous liquid. Journal of Computational Physics 222 (2), 769-795.

Hua, J., Stene, J. F., Lin, P., Mar. 2008. Numerical simulation of 3D bubbles rising in viscous liquids using a front tracking method. Journal of Computational Physics 227 (6), $3358-3382$.

Inamuro, T., Ogata, T., Tajima, S., Konishi, N., Aug. 2004. A lattice Boltzmann method for incompressible two-phase flows with large density differences. Journal of Computational Physics 198 (2), 628-644.

Jin, K., Kumar, P., Vanka, S. P., Thomas, B. G., submitted. Rise of an argon bubble in liquid steel in the presence of a transverse magnetic field.

Keshavarzi, G., Pawell, R. S., Barber, T. J., Yeoh, G. H., Jun. 2014. Transient analysis of a single rising bubble used for numerical validation for multiphase flow. Chemical Engineering Science 112, 25-34. 
Krishna, R., Urseanu, M., Van Baten, J., Ellenberger, J., 1999. Wall effects on the rise of single gas bubbles in liquids. International Communications in Heat and Mass Transfer $26(6), 781-790$.

Lalanne, B., Tanguy, S., Risso, F., 2013. Effect of rising motion on the damped shape oscillations of drops and bubbles. Physics of Fluids 25 (11), 112107.

Legendre, D., Zenit, R., Velez-Cordero, J. R., 2012. On the deformation of gas bubbles in liquids. Physics of Fluids 24 (4), 043303.

Levich, V., 1962. Physicochemical Hydrodynamics. Prentice-Hall, Englewood Cliffs, NJ.

Li, J., 1995. Calcul d'Interface Affine par Morceaux(piecewise linear interface calculation). C. R. Acad. Sci. Paris 320 (8), 391-396.

Li, K.-W. K., Schneider, A., 1993. Rise velocities of large bubbles in viscous Newtonian liquids. Journal of the American Ceramic Society 76 (1), 241-244.

Maldonado, M., Quinn, J. J., Gomez, C. O., Finch, J. A., 2013. An experimental study examining the relationship between bubble shape and rise velocity. Chemical Engineering Science 98, 7-11.

Moore, D. W., Mar. 1959. The rise of a gas bubble in a viscous liquid. Journal of Fluid Mechanics 6 (01), 113-130.

Noh, W., Woodward, P., 1976. SLIC (simple line interface calculation). In: Proceedings of the Fifth International Conference. Springer-Verlag, Berlin, pp. 330-340.

Ohta, M., Imura, T., Yoshida, Y., Sussman, M., Feb. 2005. A computational study of the effect of initial bubble conditions on the motion of a gas bubble rising in viscous liquids. International Journal of Multiphase Flow 31 (2), 223-237.

Ohta, M., Tsuji, M., Yoshida, Y., Sussman, M., Sep. 2008. The transient dynamics of a small bubble rising in a low morton number regime. Chemical Engineering \& Technology 31 (9), $1350-1357$.

Osher, S., Fedkiw, R., 2003. Level Set Methods and Dynamic Implicit Surfaces. Vol. 153. Springer New York. 
Osher, S., Sethian, J. A., 1988. Fronts propagating with curvature-dependent speed: Algorithms based on hamilton-jacobi formulations. Journal of Computational Physics 79 (1), $12-49$.

Ravelet, F., Colin, C., Risso, F., 2011. On the dynamics and breakup of a bubble rising in a turbulent flow. Physics of Fluids 23 (10), 103301.

Rider, W. J., Kothe, D. B., Apr. 1998. Reconstructing volume tracking. Journal of Computational Physics 141 (2), 112-152.

Roig, V., Roudet, M., Risso, F., Billet, A.-M., Aug. 2012. Dynamics of a high-Reynoldsnumber bubble rising within a thin gap. Journal of Fluid Mechanics 707, 444-466.

Rosenberg, B., 1950. The Drag and Shape of Air Bubbles Moving in Liquids. Report: David W. Taylor Model Basin. Navy Department, Washington DC.

Rudman, M., Aug. 1998. A volume-tracking method for incompressible multifluid flows with large density variations. International Journal for Numerical Methods in Fluids 28 (2), 357-378.

Rybczynski, W., 1911. On the translatory motion of a fluid sphere in a viscous medium. Bull. Acad. Sci., Krakow, Series A 40, 40-46.

Sanada, T., Shirota, M., Watanabe, M., Dec. 2007. Bubble wake visualization by using photochromic dye. Chemical Engineering Science 62 (24), 7264-7273.

Shew, W. L., Poncet, S., Pinton, J. F., Nov. 2006. Force measurements on rising bubbles. Journal of Fluid Mechanics 569, 51.

Shinn, A. F., 2011. Large eddy simulations of turbulent flows on graphics processing units: application to film-cooling flows. Ph.D. thesis, University of Illinois at Urbana-Champaign.

Son, G., Jun. 2003. Efficient implementation of a coupled level-set and volume-of-fluid method for three-dimensional incompressible two-phase flows. Numerical Heat Transfer, Part B: Fundamentals 43 (6), 549-565.

Sussman, M., May 2003. A second order coupled level set and volume-of-fluid method for computing growth and collapse of vapor bubbles. Journal of Computational Physics 187 (1), 110-136. 
Sussman, M., Puckett, E. G., Aug. 2000. A Coupled Level Set and Volume-of-Fluid method for computing $3 \mathrm{~d}$ and axisymmetric incompressible two-phase flows. Journal of Computational Physics 162 (2), 301-337.

Takagi, S., Prosperetti, A., Matsumoto, Y., 1994. Drag coefficient of a gas bubble in an axisymmetric shear flow. Physics of Fluids (1994-present) 6 (9).

Tomiyama, A., Celata, G., Hosokawa, S., Yoshida, S., 2002. Terminal velocity of single bubbles in surface tension force dominant regime. International Journal of Multiphase Flow 28 (9), 1497-1519.

Tripathi, M. K., Sahu, K. C., Govindarajan, R., 2015a. Dynamics of an initially spherical bubble rising in quiescent liquid. Nature Communications 6 (6268).

Tripathi, M. K., Sahu, K. C., Karapetsas, G., Sefiane, K., Matar, O. K., 1 2015b. Nonisothermal bubble rise: non-monotonic dependence of surface tension on temperature. Journal of Fluid Mechanics 763, 82-108.

Unverdi, S. O., Tryggvason, G., May 1992. A front-tracking method for viscous, incompressible, multi-fluid flows. Journal of Computational Physics 100 (1), 25-37.

Vanka, S. P., Shinn, A. F., Sahu, K. C., 2011. Computational fluid dynamics using graphics processing units: Challenges and opportunities. In: Proceedings of the ASME 2011 International Mechanical Engineering Congress and Exposition (IMECE 2011). pp. 429-437.

Wang, Z., Tong, A. Y., Mar. 2008. Deformation and oscillations of a single gas bubble rising in a narrow vertical tube. International Journal of Thermal Sciences 47 (3), 221-228.

Wu, M., Gharib, M., 2002. Experimental studies on the shape and path of small air bubbles rising in clean water. Physics of Fluids 14 (7), L49-L52.

Yang, B., Prosperetti, A., Takagi, S., 2003. The transient rise of a bubble subject to shape or volume changes. Physics of Fluids (1994-present) 15 (9).

Yu, Z., Fan, L.-S., Jun. 2008. Direct simulation of the buoyant rise of bubbles in infinite liquid using level set method. The Canadian Journal of Chemical Engineering 86 (3), 267-275.

Zhang, L., Yang, C., Mao, Z.-S., Apr. 2008. Unsteady motion of a single bubble in highly viscous liquid and empirical correlation of drag coefficient. Chemical Engineering Science 63 (8), 2099-2106. 


\section{List of Figures}

1 Validation of results with work of Bhaga and Weber Bhaga and Weber (1981) 27

2 Grid independency study: a) comparison of the rise velocities and b) comparison of the bubble shapes. The inset in the figures shows the zoom in the relevant regions. . . . . . . . . . . . . . . . . . . . . . . 28

3 Initial state of a bubble in the liquid column . . . . . . . . . . . . . . . . 29

4 Definition of height and width of the bubble for aspect ratio calculation . . . 30

5 Transient bubble shapes for confinement ratio of 4 and $M o=0.001$. . . . . 31

6 Bubble shapes for confinement ratios of 2, 3 and 4 and $M o=0.001$ : a) $B o=1$,

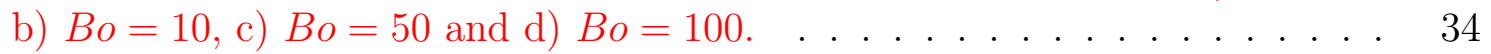

7 Rise velocities for confinement ratios of 2, 3 and 4 , and $M_{o}=0.001$ : a)

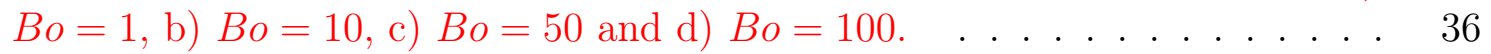

8 Aspect ratio for confinement ratios of 2, 3 and 4, and $M o=0.001$ : a) $B_{0}=1$,

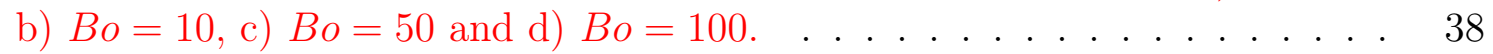

9 Bubble shapes at $t=9,9.5$ and 10 overlaid over each other for $B o=10$ and $B o=50$ and $C R=4 \ldots \ldots \ldots \ldots$

10 Instantaneous streamlines at $t=10$ for confinement ratios of 2,3 and 4 ,

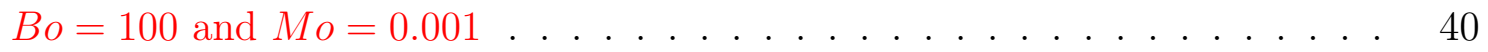

12 Top view of $z$-vorticity iso-surfaces for three confinement ratios, Bo $=100$ and $\mathrm{Mo}=0.001$. The blue and yellow colors in (a), (b) and (c) represent $\omega_{z}=-0.05$ and $\omega_{z}=0.05$ respectively. The blue and yellow colors in (a), (b) and (c) represent $\omega_{z}=-0.5$ and $\omega_{z}=0.5$ respectively. . . . . . . . . . .

13 Instantaneous streamlines at $t=10$ for confinement ratio of $4, B o=1,10,50,100$

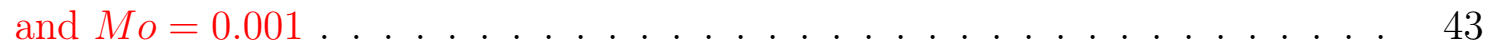

14 Transient bubble shapes for confinement ratio of 4 and $M o=0.01 \ldots$. . . . 44

15 Bubble shapes for confinement ratios of 2, 3 and 4 and $M o=0.01$ : a) $B o=1$, b) $B o=10$, c) $B o=50$ and d) $B o=100 . \ldots \ldots$. . . . . . . . . 47

16 Rise velocities for confinement ratios of 2, 3 and 4, and $M o=0.01$ : a) $B o=1$, b) $B o=10$, c) $B o=50$ and d) $B o=100 . \ldots \ldots \ldots$

17 Aspect ratio for confinement ratios of 2, 3 and 4, and $M o=0.01$ : a) $B_{o}=1$, b) $B o=10$, c) $B o=50$ and d) $B o=100 \ldots \ldots \ldots \ldots$ 


\begin{tabular}{|c|c|c|c|c|}
\hline $\begin{array}{l}\text { Test } \\
\text { case }\end{array}$ & $\begin{array}{c}\text { Test } \\
\text { conditions }\end{array}$ & $\begin{array}{c}\text { Experimentally } \\
\text { observed terminal } \\
\text { bubble shape }\end{array}$ & $\begin{array}{c}\text { Numerically } \\
\text { predicted } \\
\text { terminal bubble } \\
\text { shape }\end{array}$ & $\begin{array}{c}\text { Simulation } \\
\text { conditions and } \\
\text { predicted } \\
\text { terminal Reynolds } \\
\text { number } \\
\end{array}$ \\
\hline $\mathbf{A}$ & $\begin{array}{c}\mathrm{Bo}=17.7 \\
\mathrm{Mo}=711 \\
\mathbf{R e}=\mathbf{0 . 2 3 2}\end{array}$ & & & $\begin{array}{c}\mathrm{Bo}=17.7 \\
\mathrm{Mo}=711 \\
\mathbf{R e}=\mathbf{0 . 2 2 7}\end{array}$ \\
\hline B & $\begin{array}{c}\mathrm{Bo}=243 \\
\mathrm{Mo}=266 \\
\mathbf{R e}=\mathbf{7 . 7 7}\end{array}$ & & & $\begin{array}{c}\mathrm{Bo}=243 \\
\mathrm{Mo}=266 \\
\mathbf{R e}=\mathbf{7 . 6 1}\end{array}$ \\
\hline
\end{tabular}

Fig. 1: Validation of results with work of Bhaga and Weber Bhaga and Weber (1981) 


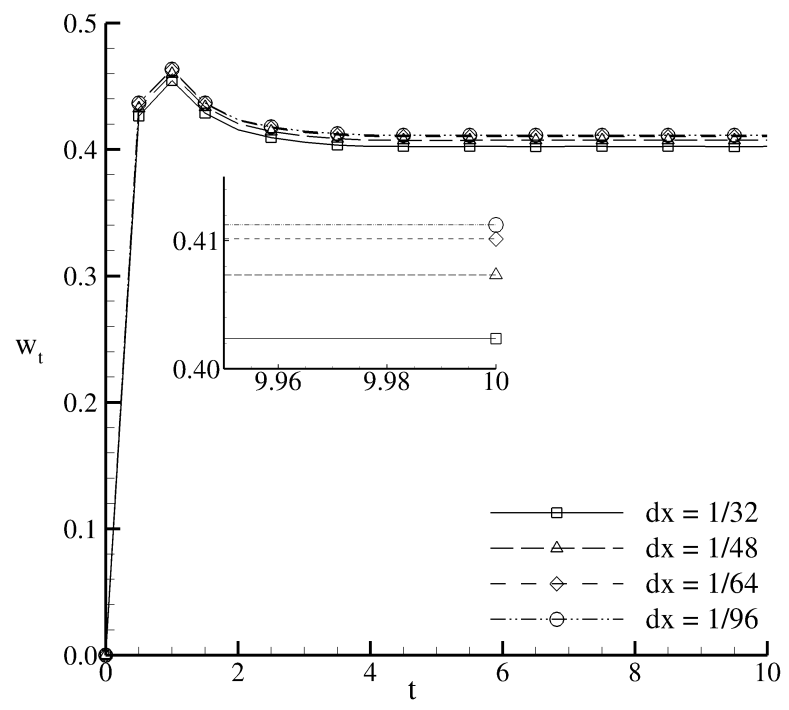

(a)

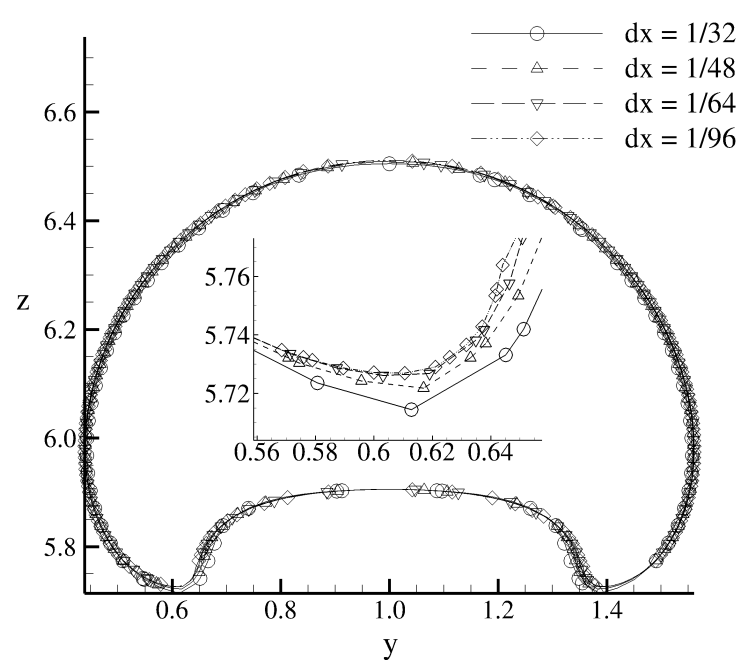

(b)

Fig. 2: Grid independency study: a) comparison of the rise velocities and b) comparison of the bubble shapes. The inset in the figures shows the zoom in the relevant regions. 


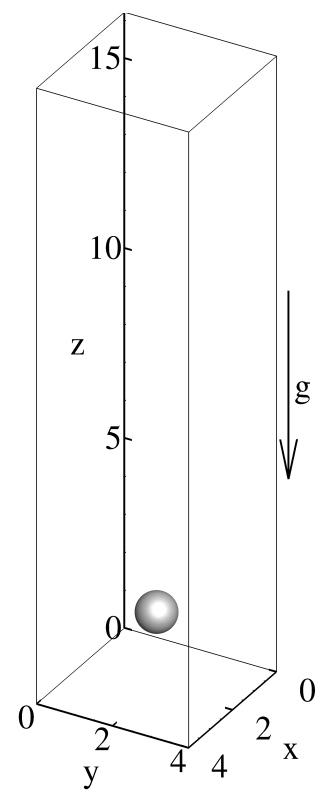

Fig. 3: Initial state of a bubble in the liquid column 


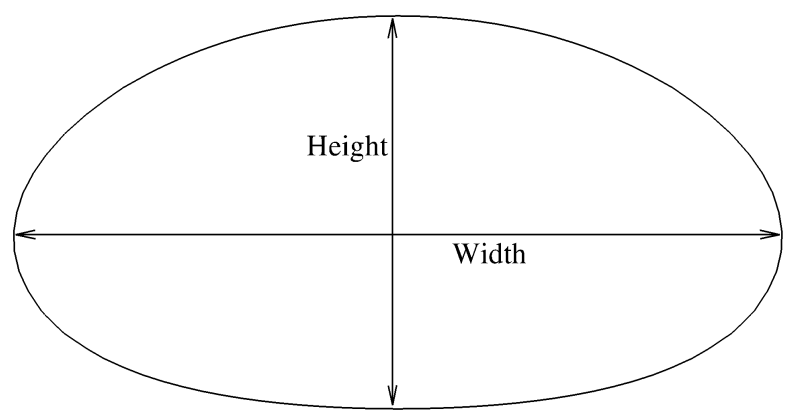

Fig. 4: Definition of height and width of the bubble for aspect ratio calculation 


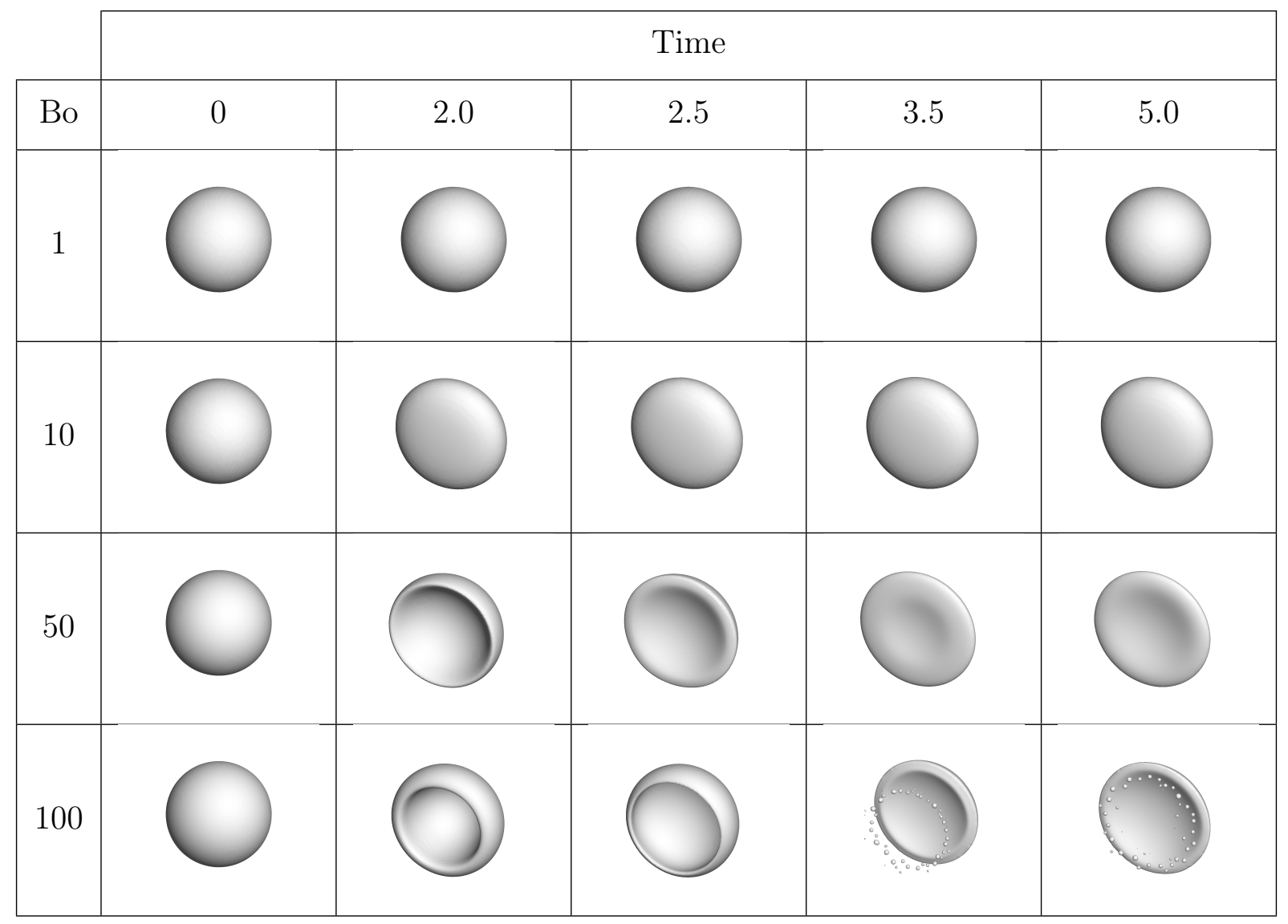

Fig. 5: Transient bubble shapes for confinement ratio of 4 and $M o=0.001$. 

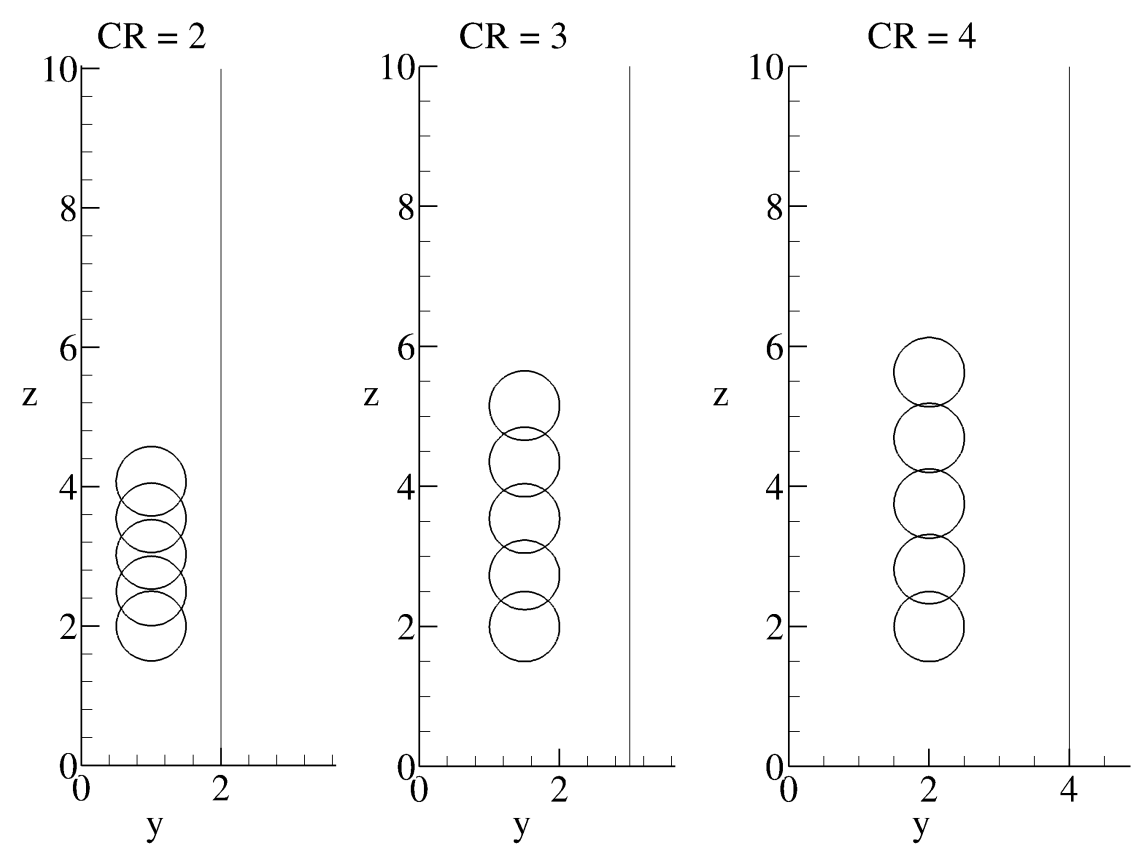

(a) Bubble shapes at $t=0,2.5,5,7.5$ and 10 for $B o=1$
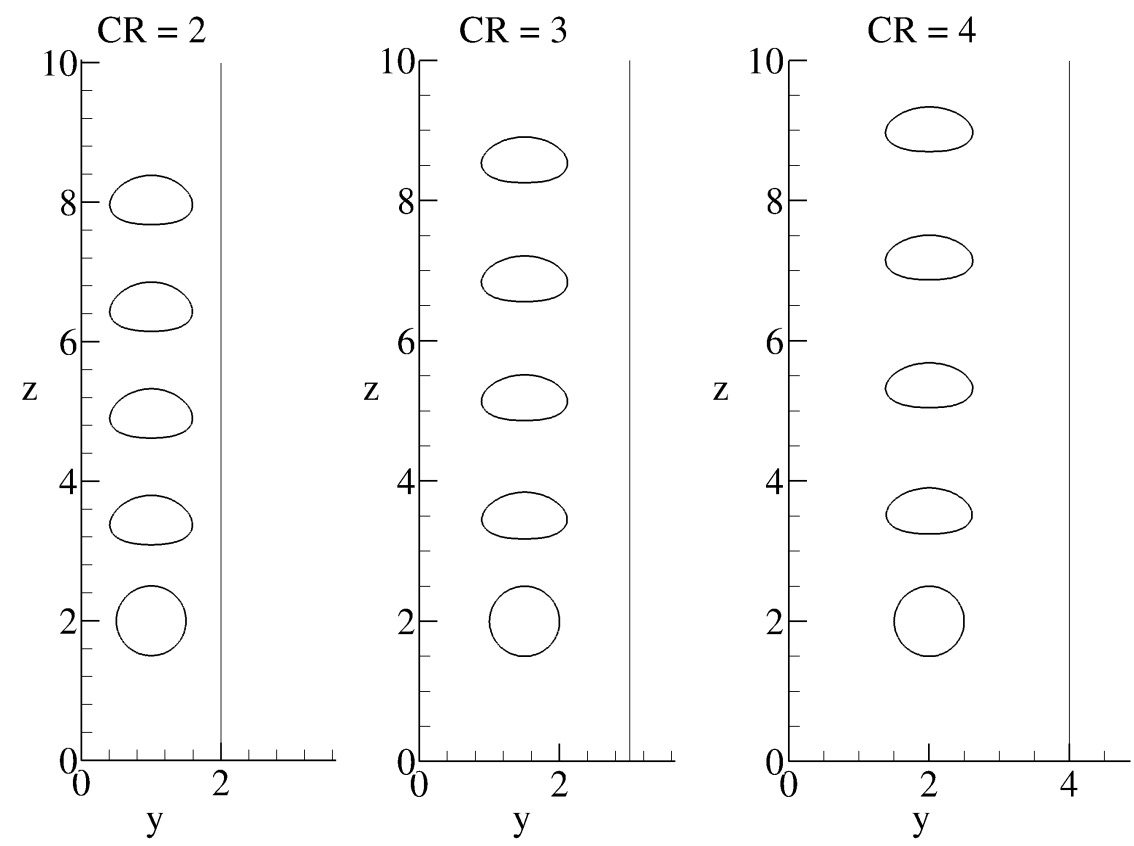

(b) Bubble shapes at $t=0,2.5,5,7.5$ and 10 for $B o=10$ 

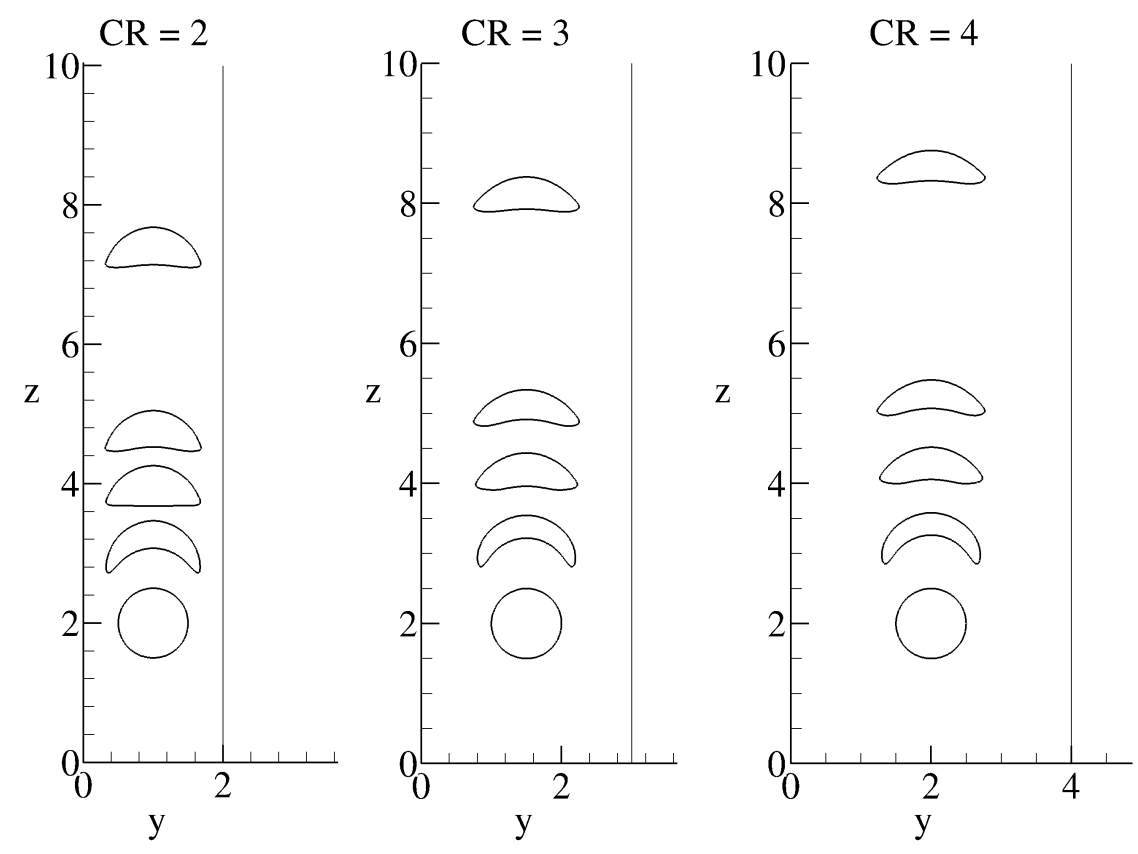

(c) Bubble shapes at $t=0,2.5,5,7.5$ and 10 for $B o=50$ 

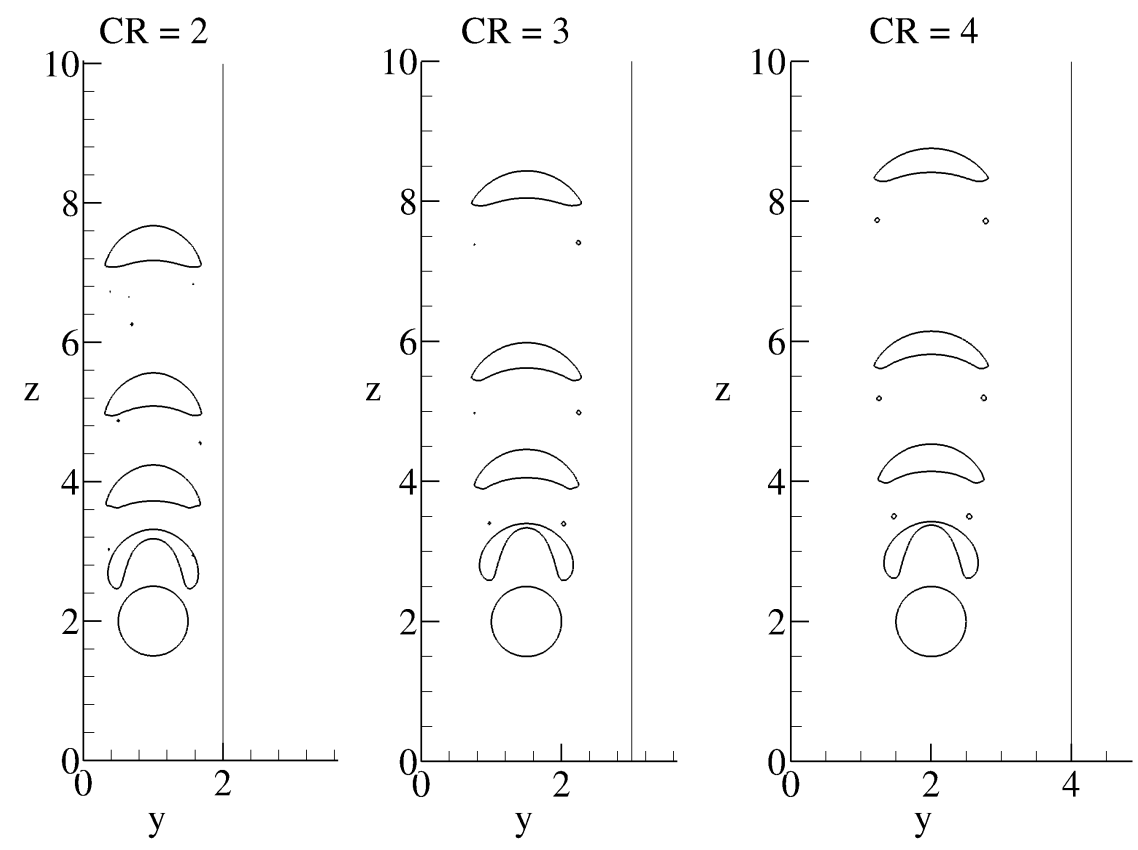

(d) Bubble shapes at $t=0,2.5,5,7.5$ and 10 for $B o=100$

Fig. 6: Bubble shapes for confinement ratios of 2, 3 and 4 and $M o=0.001$ : a) $B o=1$, b) $B o=10$, c) $B o=50$ and d) $B o=100$. 


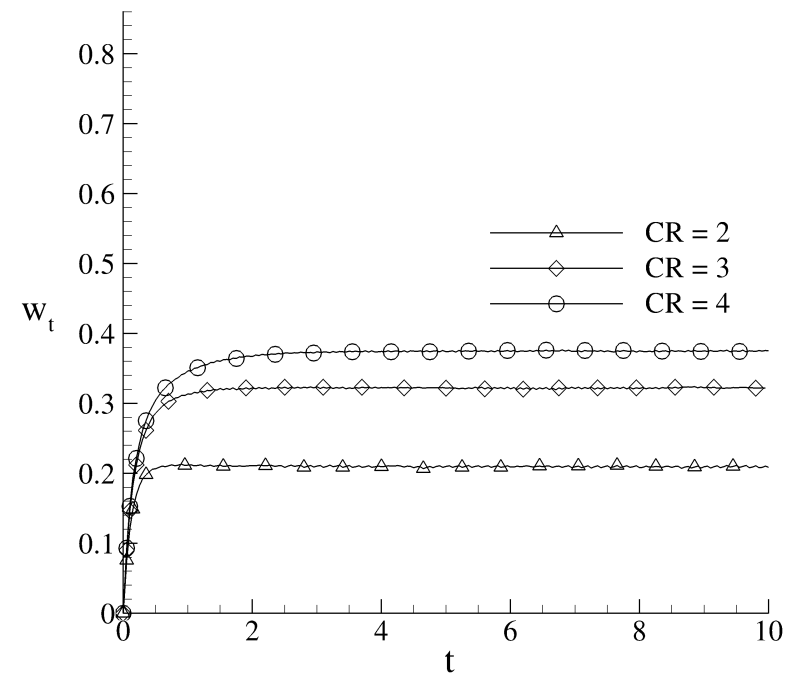

(a) Bo $=1$

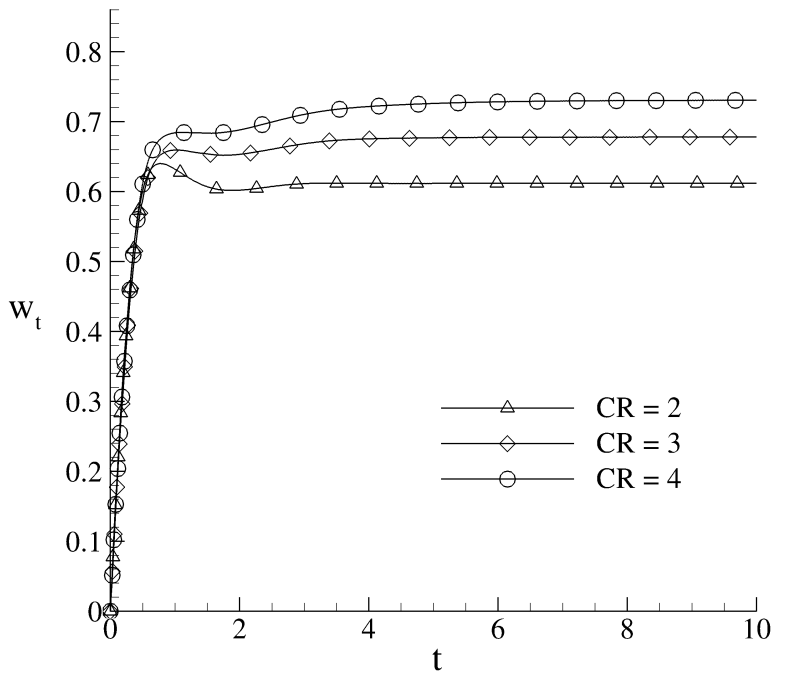

(b) $\mathrm{Bo}=10$ 


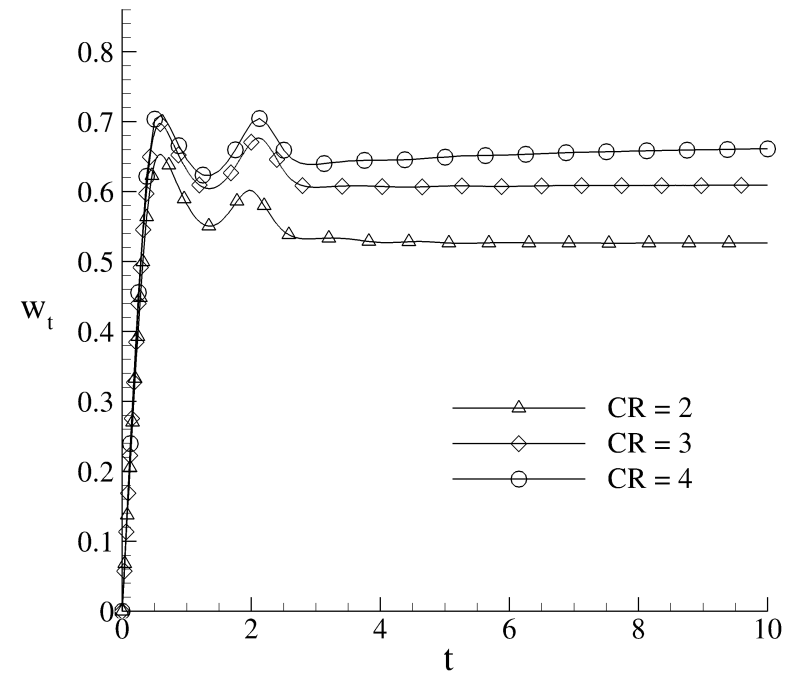

(c) $\mathrm{Bo}=50$

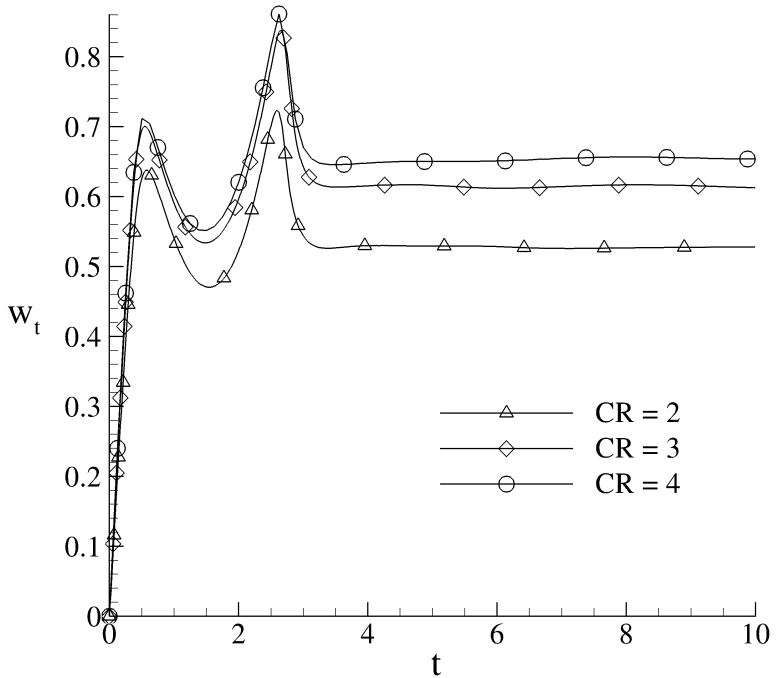

(d) Bo $=100$

Fig. 7: Rise velocities for confinement ratios of 2, 3 and 4, and $M o=0.001$ : a) $B o=1$, b) $B o=10$, c) $B o=50$ and d) $B o=100$. 


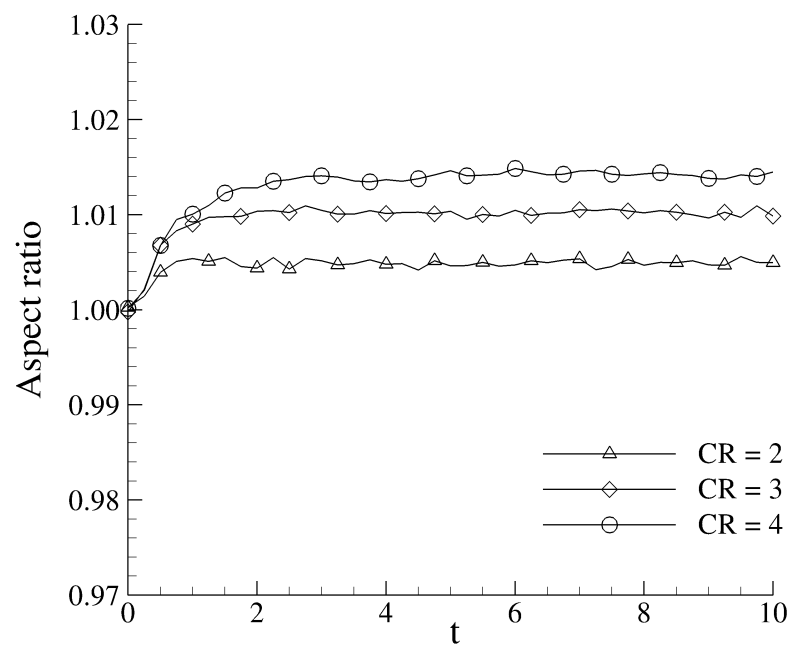

(a) $\mathrm{Bo}=1$

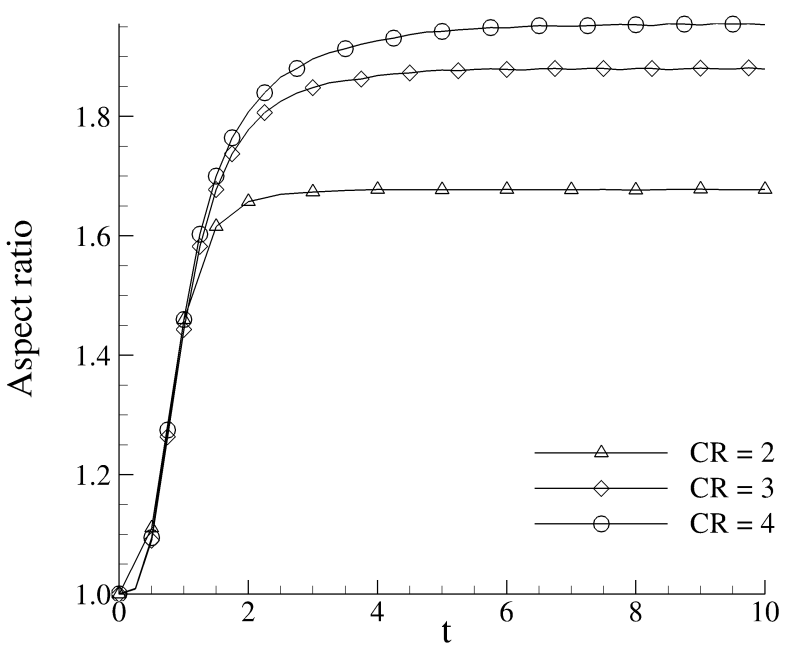

(b) $\mathrm{Bo}=10$ 


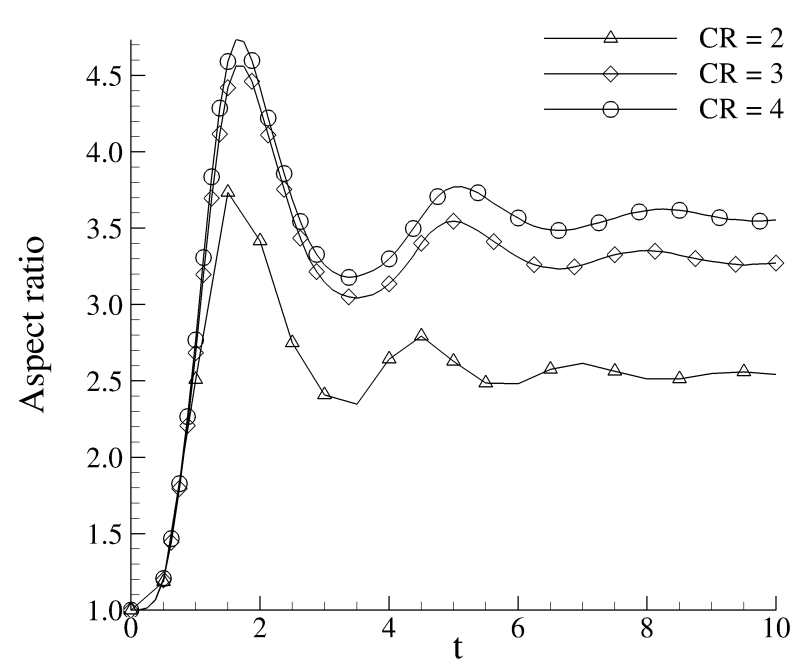

(c) Bo $=50$

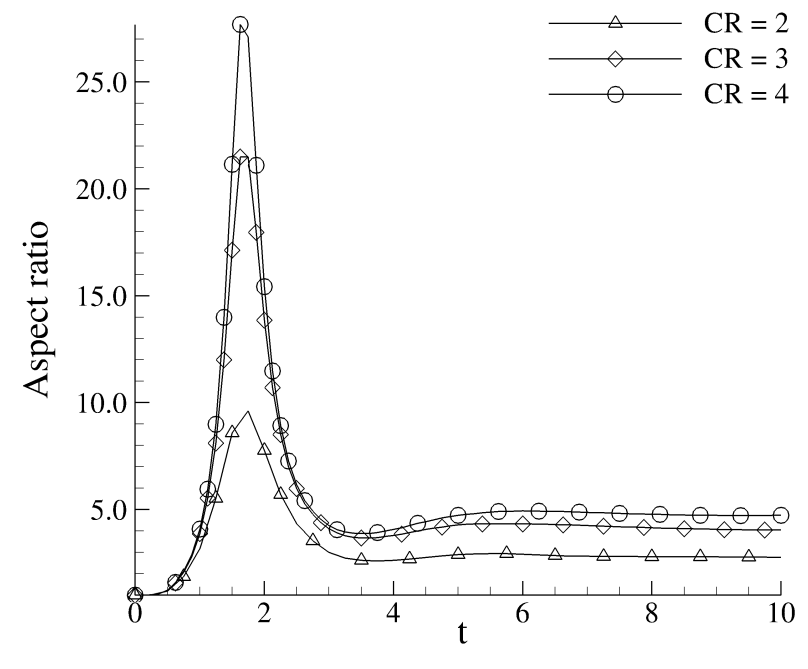

(d) Bo $=100$

Fig. 8: Aspect ratio for confinement ratios of 2, 3 and 4, and $M o=0.001$ : a) $B o=1$, b) $B o=10$, c) $B o=50$ and d) $B o=100$. 


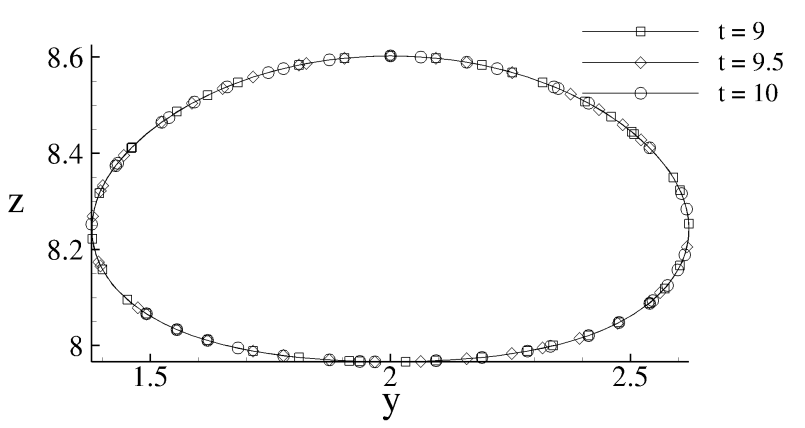

(a) $\mathrm{Bo}=10$

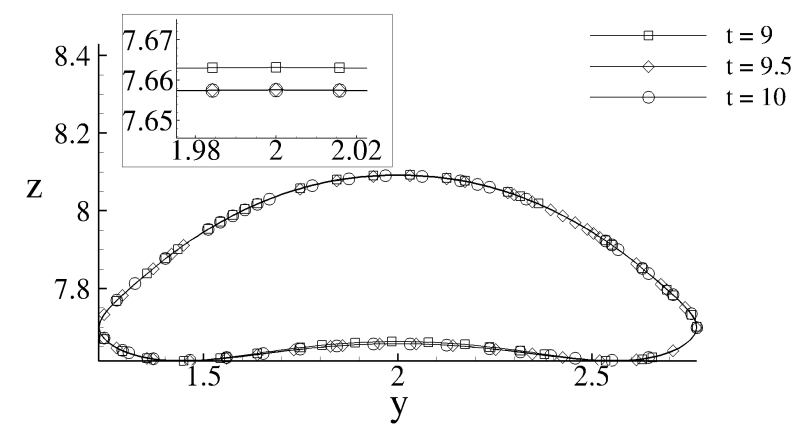

(b) Bo $=50$

Fig. 9: Bubble shapes at $t=9,9.5$ and 10 overlaid over each other for $B o=10$ and $B o=50$ and $C R=4$. 

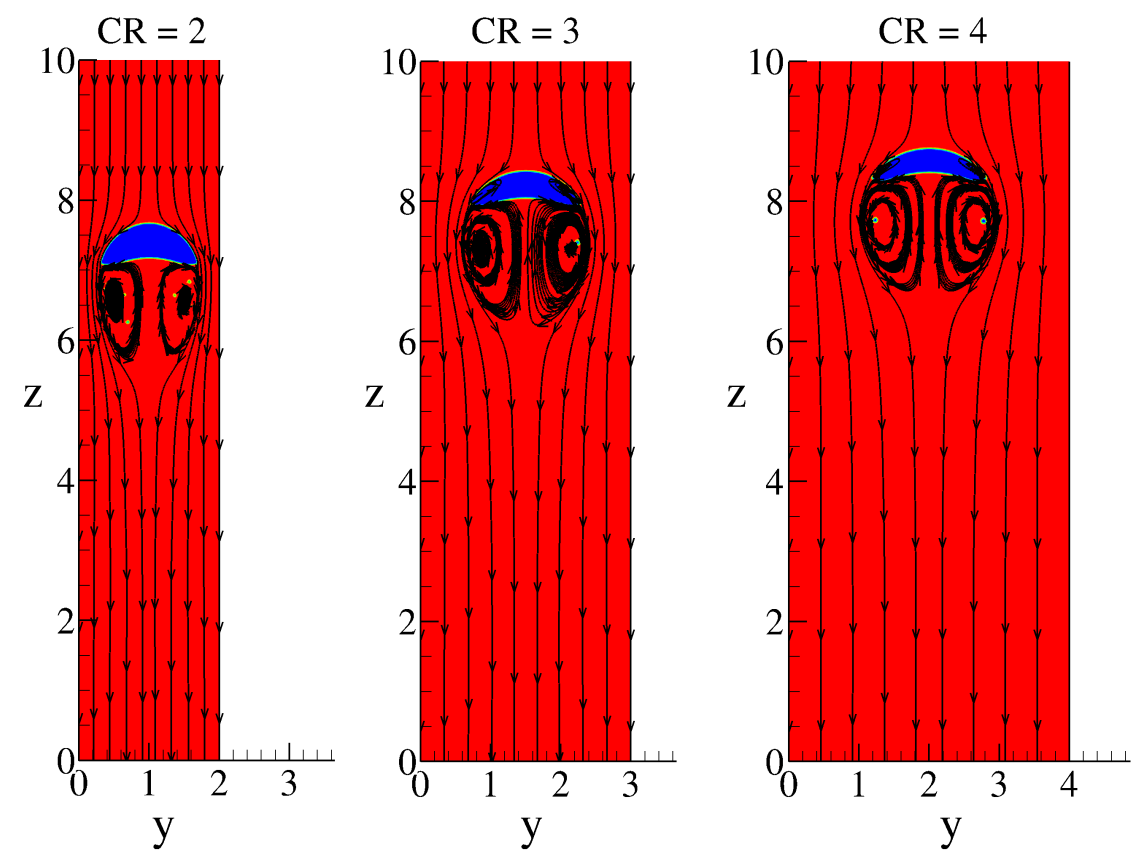

Fig. 10: Instantaneous streamlines at $t=10$ for confinement ratios of 2,3 and $4, B o=100$ and $M o=0.001$ 


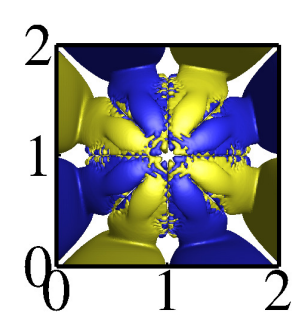

(a) $\mathrm{CR}=2$

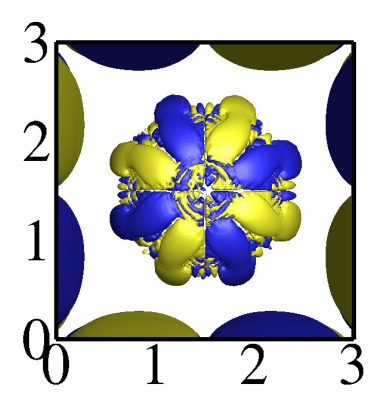

(b) $\mathrm{CR}=3$

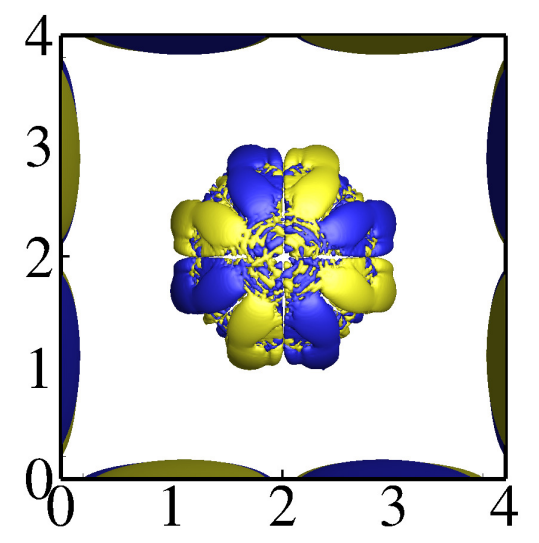

(c) $\mathrm{CR}=4$ 


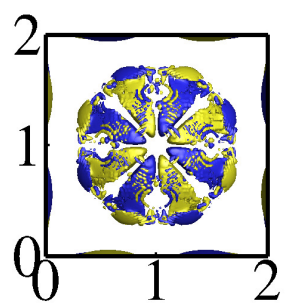

(a) $\mathrm{CR}=2$

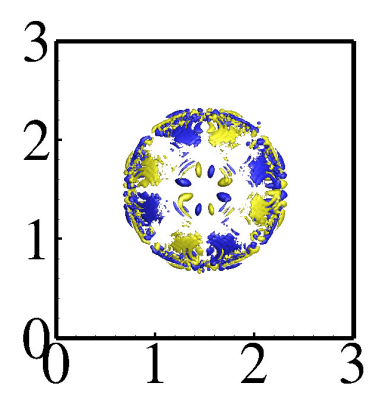

(b) $\mathrm{CR}=3$

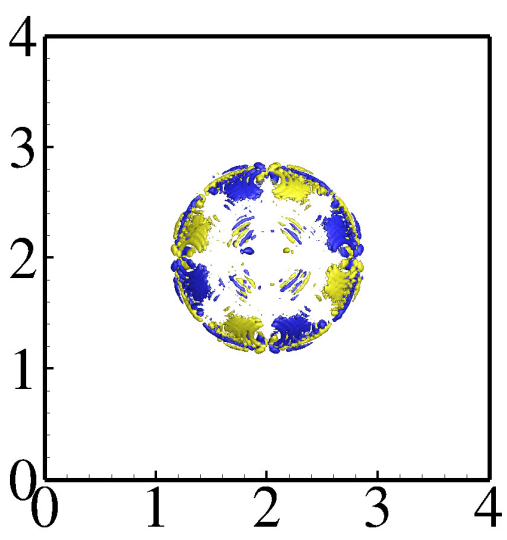

(c) $\mathrm{CR}=4$

Fig. 12: Top view of $z$-vorticity iso-surfaces for three confinement ratios, $\mathrm{Bo}=100$ and $\mathrm{Mo}=0.001$. The blue and yellow colors in (a), (b) and (c) represent $\omega_{z}=-0.05$ and $\omega_{z}=0.05$ respectively. The blue and yellow colors in (a), (b) and (c) represent $\omega_{z}=-0.5$ and $\omega_{z}=0.5$ respectively. 

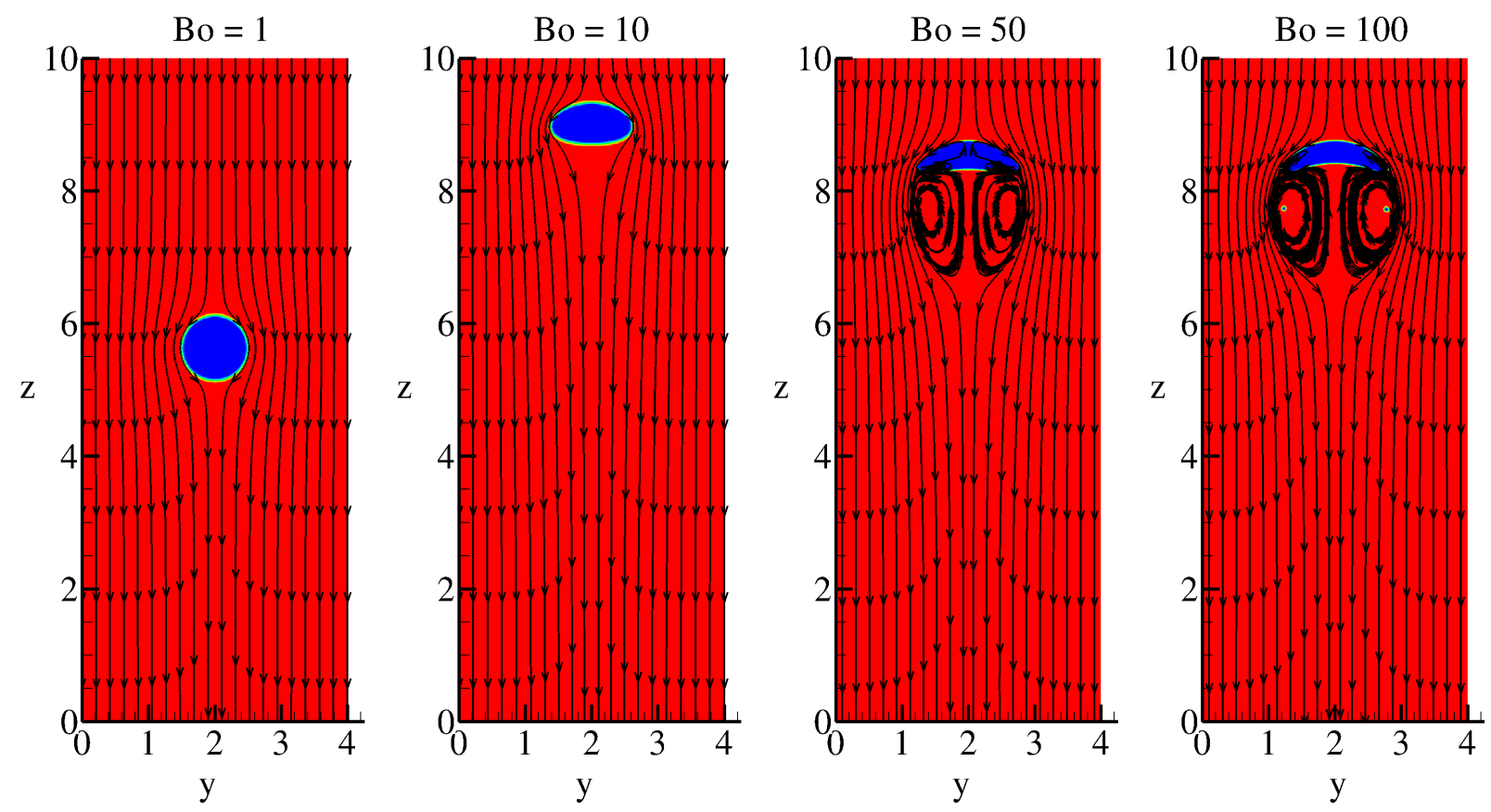

Fig. 13: Instantaneous streamlines at $t=10$ for confinement ratio of $4, B o=1,10,50,100$ and $M o=0.001$ 


\begin{tabular}{|l|l|l|l|l|l|}
\cline { 2 - 6 } \multicolumn{1}{|c|}{ Bo } & 0 & 2.0 & 2.5 & 3.5 & 5.0 \\
\hline 1 & & & & & \\
\hline
\end{tabular}

Fig. 14: Transient bubble shapes for confinement ratio of 4 and $M o=0.01$. 

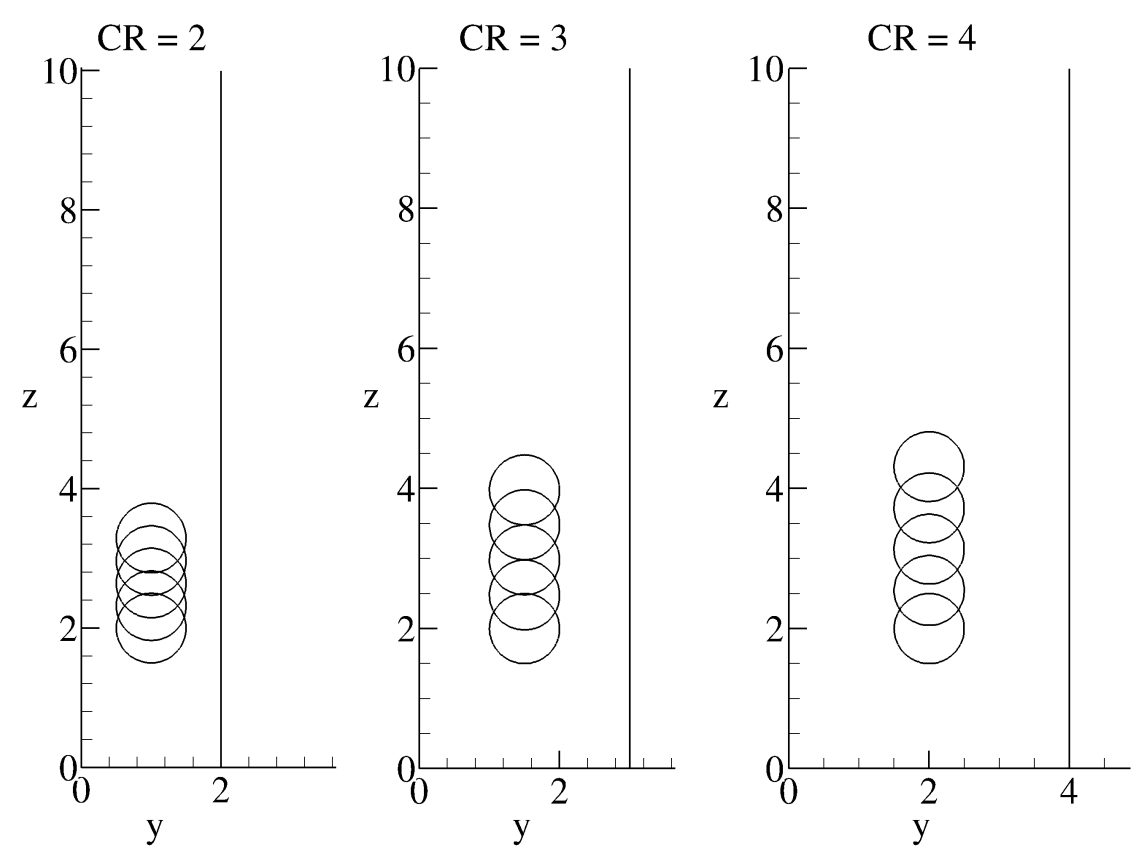

(a) Bubble shapes at $t=0,2.5,5,7.5$ and 10 for $B o=1$
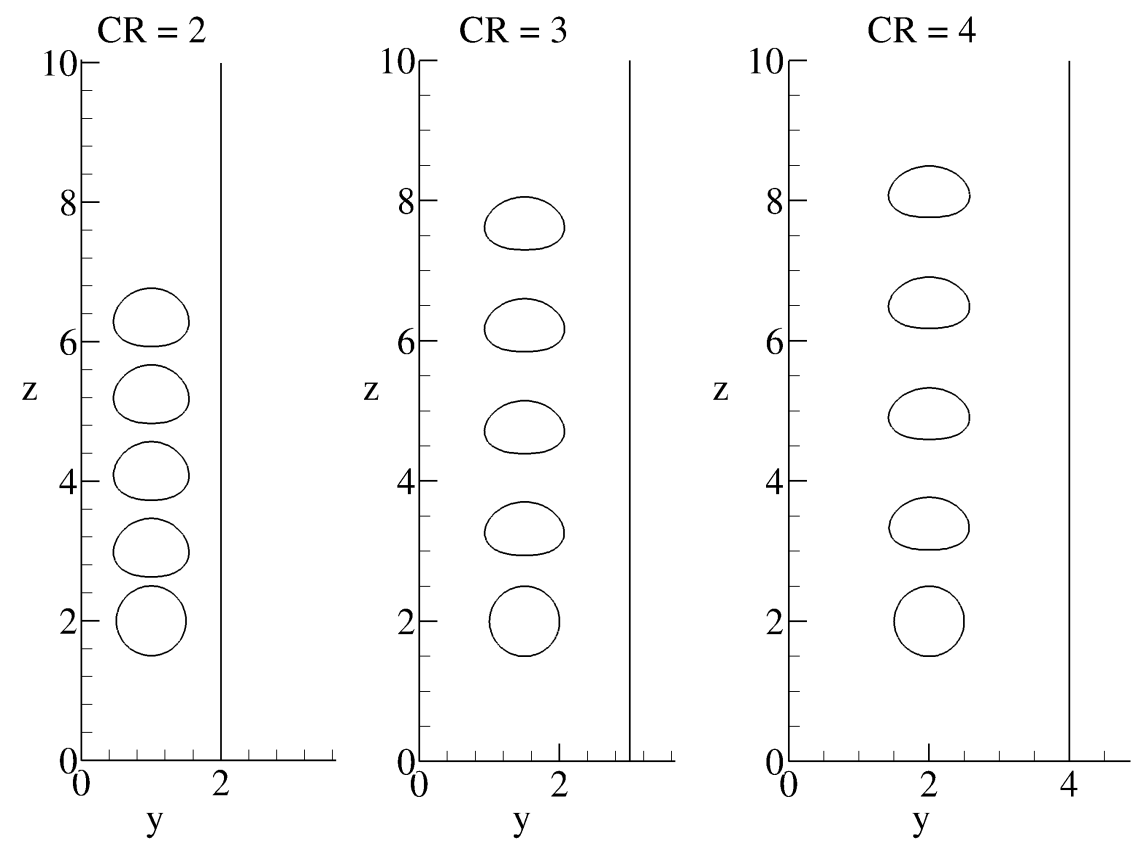

(b) Bubble shapes at $t=0,2.5,5,7.5$ and 10 for $B o=10$ 

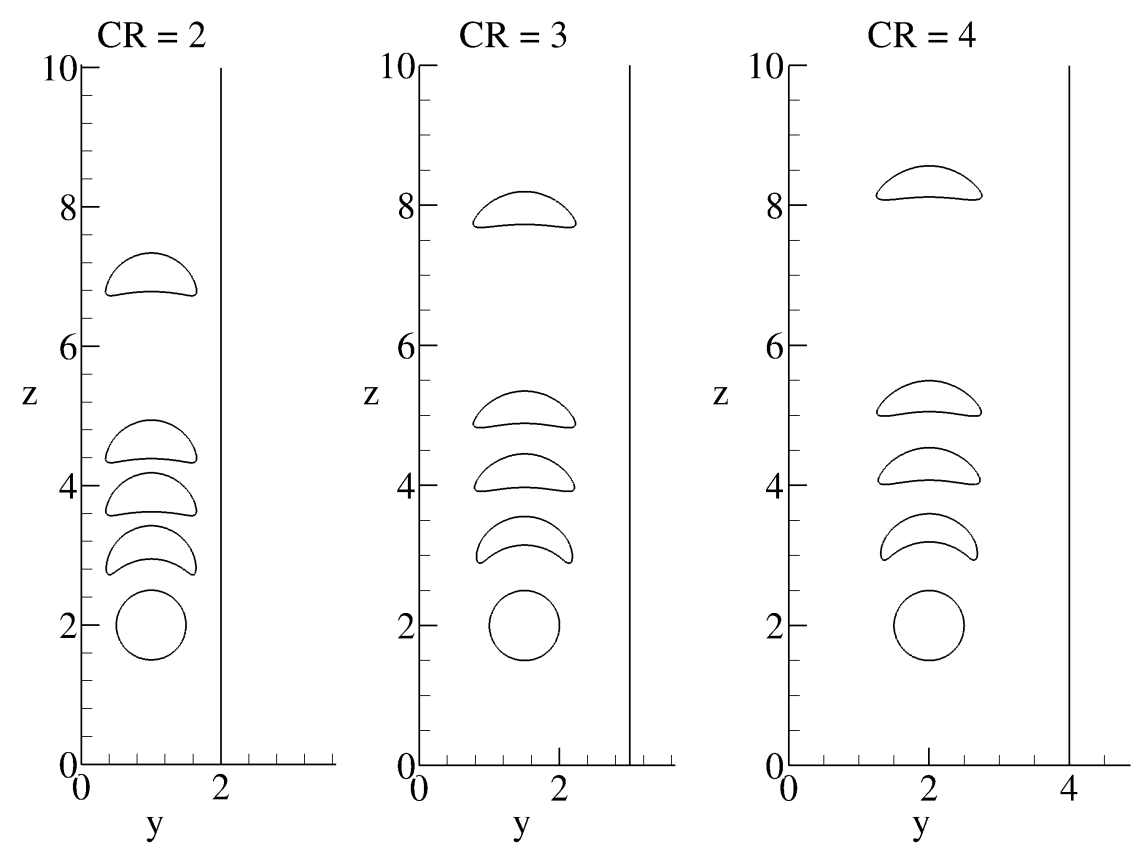

(c) Bubble shapes at $t=0,2.5,5,7.5$ and 10 for $B o=50$ 

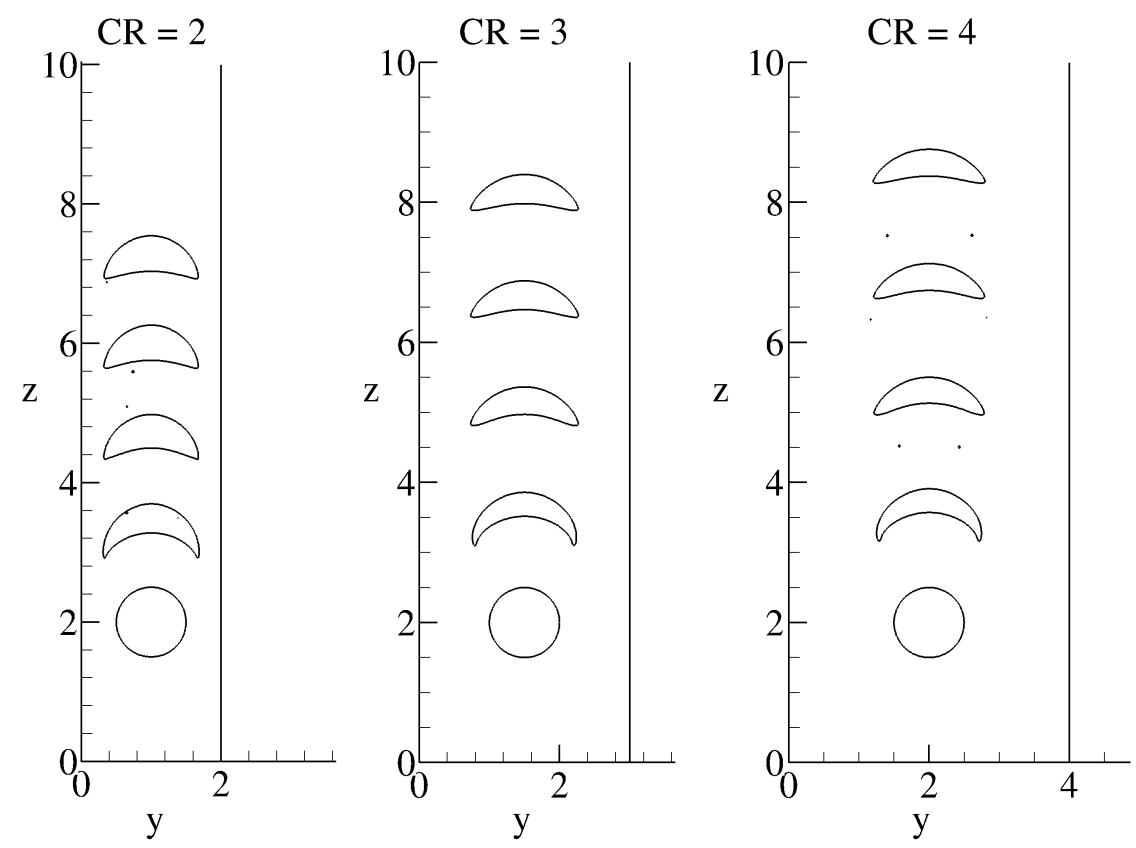

(d) Bubble shapes at $t=0,2.5,5,7.5$ and 10 for $B o=100$

Fig. 15: Bubble shapes for confinement ratios of 2, 3 and 4 and $M o=0.01$ : a) $B o=1$, b) $B o=10$, c) $B o=50$ and d) $B o=100$. 


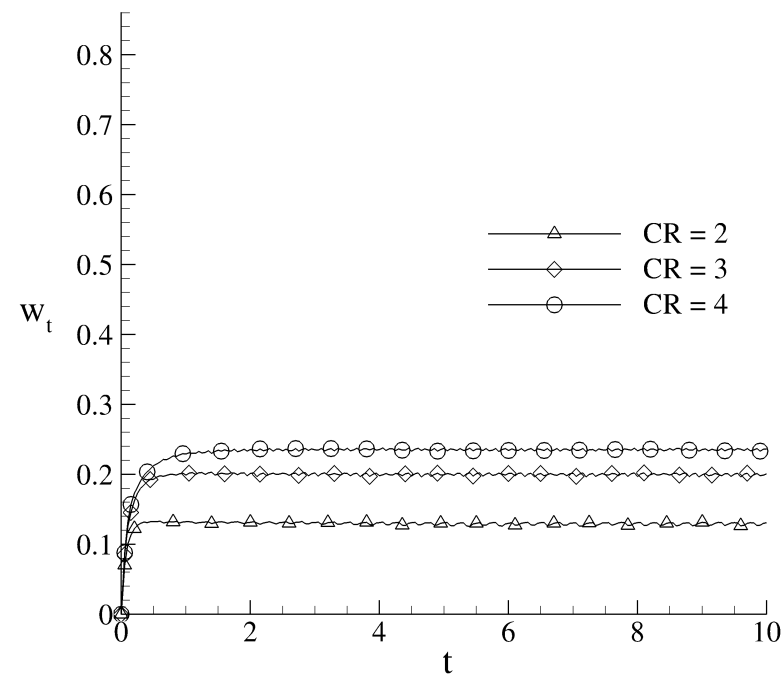

(a) Bo $=1$

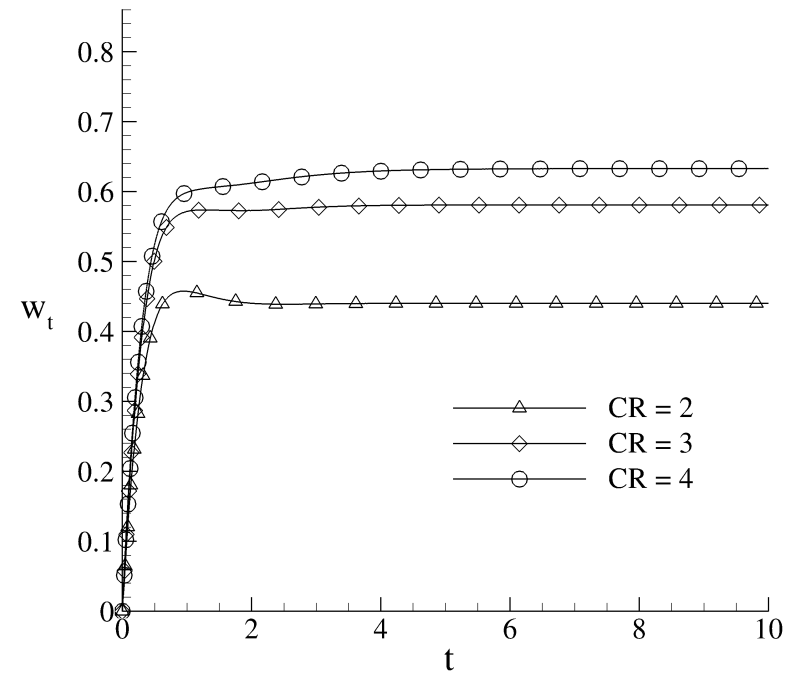

(b) $\mathrm{Bo}=10$ 


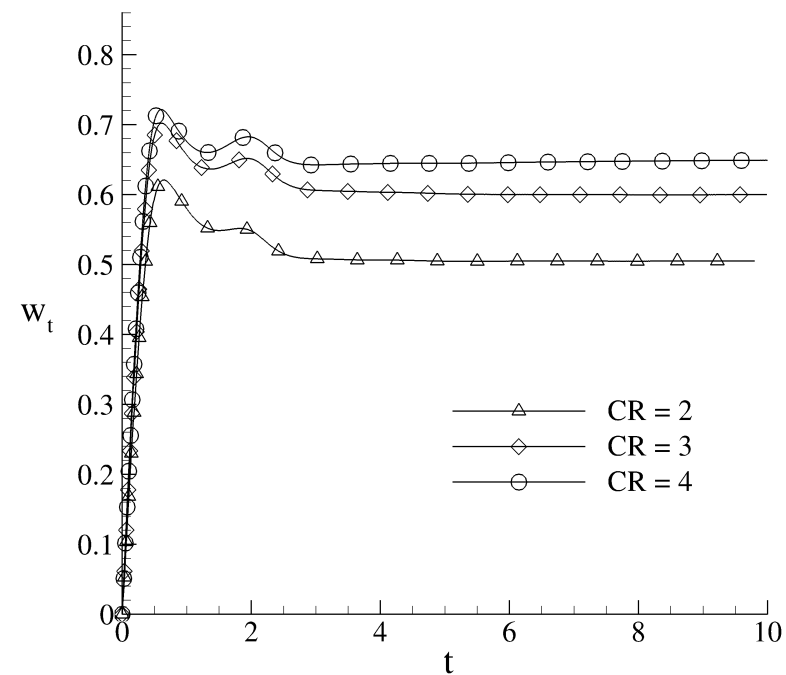

(c) $\mathrm{Bo}=50$

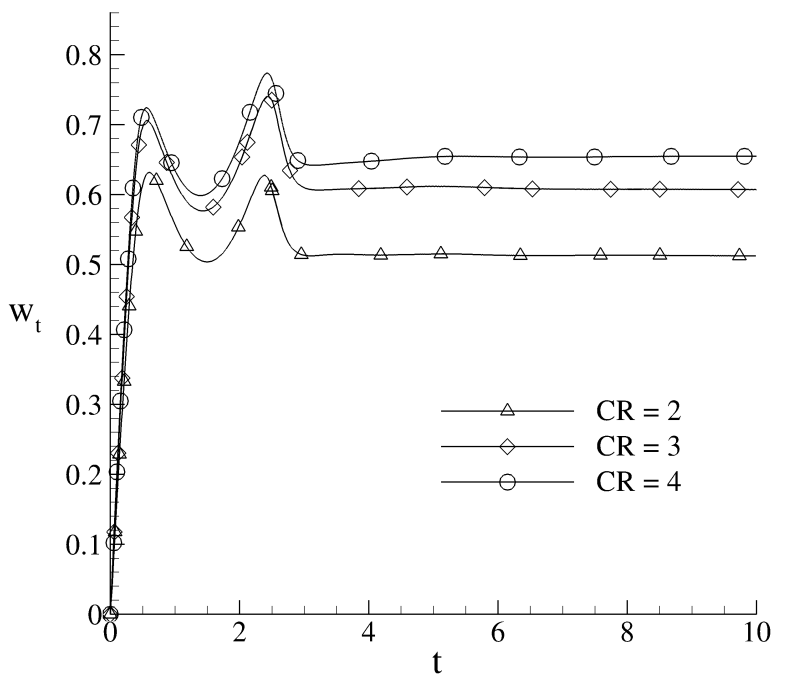

(d) Bo $=100$

Fig. 16: Rise velocities for confinement ratios of 2, 3 and 4, and $M o=0.01$ : a) $B o=1$, b) $B o=10$, c) $B o=50$ and d) $B o=100$. 


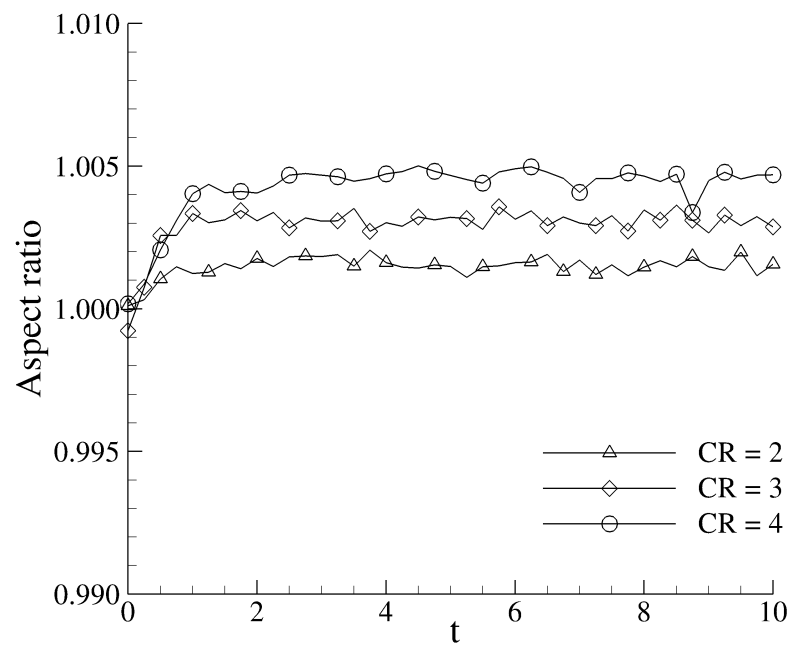

(a) Bo $=1$

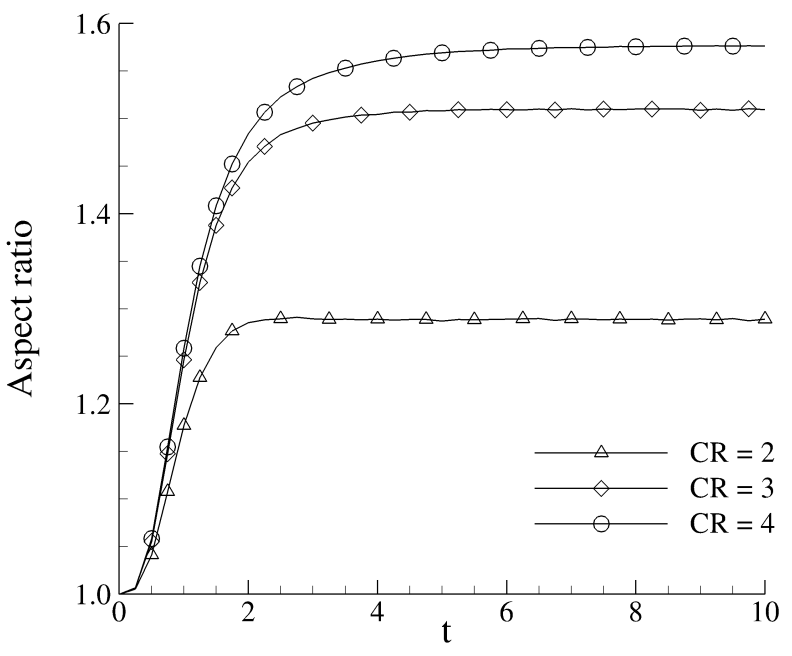

(b) $\mathrm{Bo}=10$ 


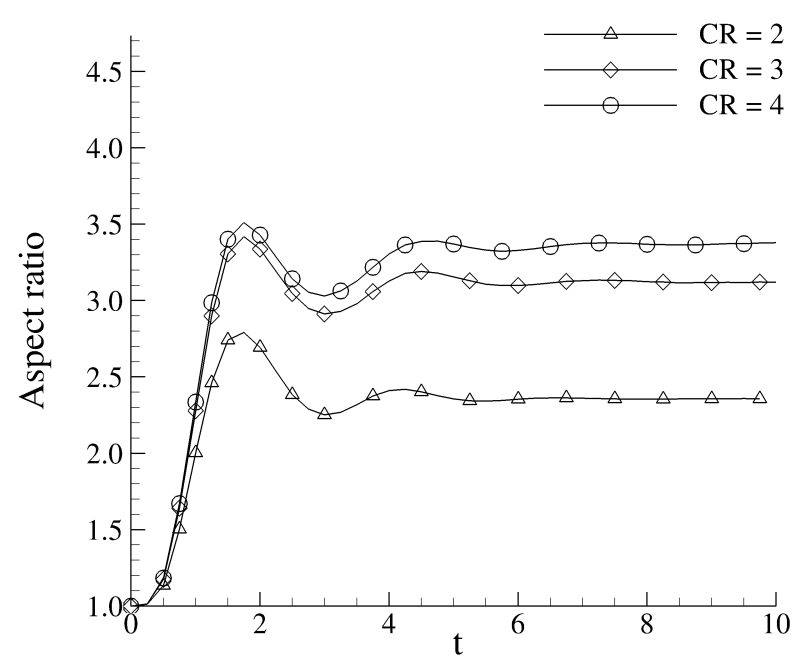

(c) Bo $=50$

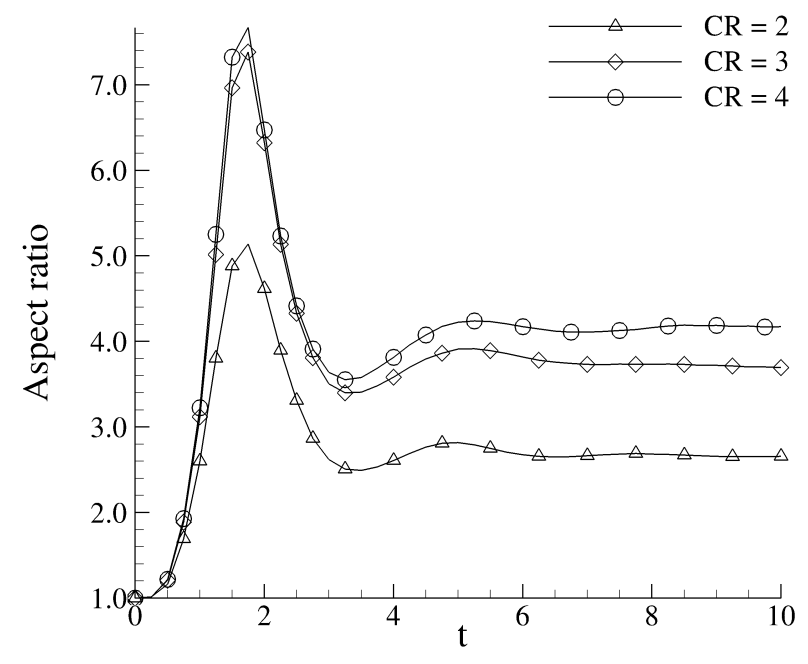

(d) Bo $=100$

Fig. 17: Aspect ratio for confinement ratios of 2, 3 and 4, and $M o=0.01$ : a) $B o=1$, b) $B o=10$, c) $B o=50$ and d) $B o=100$. 


\section{List of Tables}

1 Fluid properties ......................... 53

2 Terminal Reynolds number . . . . . . . . . . . . . . . . . . . 54 
Tab. 1: Fluid properties

\begin{tabular}{|c|c|c|c|c|c|}
\hline $\begin{array}{c}\text { Morton } \\
\text { number }\end{array}$ & $\rho_{l}\left(\mathrm{~kg} / \mathrm{m}^{3}\right)$ & $\rho_{g}\left(\mathrm{~kg} / \mathrm{m}^{3}\right)$ & $\mu_{l}($ Pa.s $)$ & $\mu_{g}($ Pa.s $)$ & $\sigma(\mathrm{mN} / \mathrm{m})$ \\
\hline 0.001 & 1215 & 1.184 & $\begin{array}{c}7.506 \times \\
10^{-2}\end{array}$ & $\begin{array}{c}1.824 \times \\
10^{-5}\end{array}$ & 63.5 \\
\hline 0.01 & 1215 & 1.184 & $\begin{array}{c}13.34 \times \\
10^{-2}\end{array}$ & $\begin{array}{c}1.824 \times \\
10^{-5}\end{array}$ & 63.5 \\
\hline
\end{tabular}


Tab. 2: Terminal Reynolds number

\begin{tabular}{|c|c|c|c|c|c|c|}
\hline \multirow{2}{*}{$\begin{array}{c}\text { Bond } \\
(B)\end{array}$} & \multicolumn{5}{|c|}{ Confinement Ratio (CR) } \\
\cline { 2 - 7 } & \multicolumn{2}{|c|}{$\mathbf{2}$} & \multicolumn{2}{|c|}{$\mathbf{3}$} & \multicolumn{2}{|c|}{$\mathbf{4}$} \\
\cline { 2 - 7 } & \multicolumn{2}{|c|}{ Morton Number $(M o)$} & \multicolumn{2}{|c|}{ Morton Number $(M o)$} & \multicolumn{2}{|c|}{ Morton Number $(M o)$} \\
\cline { 2 - 7 } & $\mathbf{0 . 0 0 1}$ & $\mathbf{0 . 0 1}$ & $\mathbf{0 . 0 0 1}$ & $\mathbf{0 . 0 1}$ & $\mathbf{0 . 0 0 1}$ & $\mathbf{0 . 0 1}$ \\
\hline $\mathbf{1}$ & 1.18 & 0.42 & 1.81 & 0.63 & 2.11 & 0.75 \\
\hline $\mathbf{1 0}$ & 19.34 & 7.89 & 21.44 & 10.42 & 23.10 & 11.35 \\
\hline $\mathbf{5 0}$ & 55.65 & 30.27 & 64.42 & 35.97 & 69.87 & 38.91 \\
\hline $\mathbf{1 0 0}$ & 93.83 & 51.69 & 109.12 & 61.25 & 116.29 & 66.04 \\
\hline
\end{tabular}

